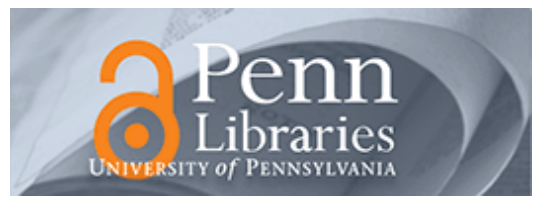

University of Pennsylvania

ScholarlyCommons

Finance Papers

Wharton Faculty Research

$5-2015$

\title{
What is the Chance That the Equity Premium Varies Over Time? Evidence From Regressions on the Dividend-Price Ratio
}

Jessica A. Wachter

University of Pennsylvania

Missaka Warusawitharana

Follow this and additional works at: https://repository.upenn.edu/fnce_papers

Part of the Finance Commons, and the Finance and Financial Management Commons

\section{Recommended Citation}

Wachter, J. A., \& Warusawitharana, M. (2015). What is the Chance That the Equity Premium Varies Over Time? Evidence From Regressions on the Dividend-Price Ratio. Journal of Econometrics, 186 (1), 74-93. http://dx.doi.org/10.1016/j.jeconom.2014.05.018

This paper is posted at ScholarlyCommons. https://repository.upenn.edu/fnce_papers/210

For more information, please contact repository@pobox.upenn.edu. 


\title{
What is the Chance That the Equity Premium Varies Over Time? Evidence From Regressions on the Dividend-Price Ratio
}

\author{
Abstract

\section{Keywords} \\ return predictability, bayesian statistics, model uncertainty \\ Disciplines \\ Economics | Finance | Finance and Financial Management
}

We examine the evidence on excess stock return predictability in a Bayesian setting in which the investor faces uncertainty about both the existence and strength of predictability. When we apply our methods to the dividend-price ratio, we find that even investors who are quite skeptical about the existence of predictability sharply modify their views in favor of predictability when confronted by the historical time series of returns and predictor variables. Correctly taking into account the stochastic properties of the regressor has a dramatic impact on inference, particularly over the 2000-2005 period. 
NBER WORKING PAPER SERIES

\title{
WHAT IS THE CHANCE THAT THE EQUITY PREMIUM VARIES OVER TIME? EVIDENCE FROM REGRESSIONS ON THE DIVIDEND-PRICE RATIO
}

\author{
Jessica A. Wachter \\ Missaka Warusawitharana \\ Working Paper 17334 \\ http://www.nber.org/papers/w17334
NATIONAL BUREAU OF ECONOMIC RESEARCH
1050 Massachusetts Avenue
Cambridge, MA 02138

August 2011

This paper previously circulated under the title "What is the chance that the equity premium varies over time? Evidence from predictive regressions." We are grateful to Sean Campbell, Mark Fisher, Michael Johannes, Matthew Pritsker, Robert Stambaugh, Stijn van Nieuwerburgh, Jonathan Wright, Moto Yogo, Hao Zhou and seminar participants at the 2008 meetings of the American Finance Association, the 2007 CIRANO Financial Econometrics Conference, the 2007 Winter Meeting of the Econometric Society, the 2010 Federal Reserve Conference on Financial Markets, the Federal Reserve Board, the University of California at Berkeley and the Wharton School for helpful comments. We are grateful for financial support from the Aronson+Johnson+Ortiz fellowship through the Rodney L. White Center for Financial Research. This manuscript does not reflect the views of the Board of Governors of the Federal Reserve System, its staff, or the National Bureau of Economic Research.

NBER working papers are circulated for discussion and comment purposes. They have not been peerreviewed or been subject to the review by the NBER Board of Directors that accompanies official NBER publications.

(C) 2011 by Jessica A. Wachter and Missaka Warusawitharana. All rights reserved. Short sections of text, not to exceed two paragraphs, may be quoted without explicit permission provided that full credit, including $(\subset$ notice, is given to the source. 
What is the Chance that the Equity Premium Varies over Time? Evidence from Regressions on the Dividend-Price Ratio

Jessica A. Wachter and Missaka Warusawitharana

NBER Working Paper No. 17334

August 2011, Revised January 2014

JEL No. C11,C22,G11,G17

\begin{abstract}
We examine the evidence on excess stock return predictability in a Bayesian setting in which the investor faces uncertainty about both the existence and strength of predictability. When we apply our methods to the dividend-price ratio, we find that even investors who are quite skeptical about the existence of predictability sharply modify their views in favor of predictability when confronted by the historical time series of returns and predictor variables. Correctly taking into account the stochastic properties of the regressor has a dramatic impact on inference, particularly over the 2000-2005 period.
\end{abstract}

Jessica A. Wachter

Department of Finance

$2300 \mathrm{SH}-\mathrm{DH}$

The Wharton School

University of Pennsylvania

3620 Locust Walk

Philadelphia, PA 19104

and NBER

jwachter@wharton.upenn.edu

Missaka Warusawitharana

Department of Research and Statistics

Board of Governors of the Federal Reserve

Mail Stop 97

20th and Constitution Ave

Washington D.C., 20551

Missaka.N.Warusawitharana@frb.gov 


\section{Introduction}

In this study, we evaluate the evidence in favor of excess stock return predictability from the perspective of a Bayesian investor. We focus on the case of a single predictor variable to highlight the complex statistical issues that come into play in this deceptively simple problem.

The investor in our model considers the evidence in favor of the following linear model for excess returns:

$$
r_{t+1}=\alpha+\beta x_{t}+u_{t+1}
$$

where $r_{t+1}$ denotes the return on a broad stock index in excess of the riskfree rate, $x_{t}$ denotes a predictor variable, and $u_{t+1}$ the unpredictable component of the return. The investor also places a finite probability on the following model:

$$
r_{t+1}=\alpha+u_{t+1}
$$

Namely, the investor assigns a prior probability $q$ to the state of the world in which returns are predictable (because the prior on $\beta$ will be smooth, the chance of $\beta=0$ in (1) is infinitesimal), and a probability $1-q$ to the state of the world in which returns are completely unpredictable. In both cases, the parameters are unknown. Thus our model allows for both parameter uncertainty and "model uncertainty". ${ }^{1}$

Allowing for a non-zero probability on (2) is one way in which we depart from previous studies. Previous Bayesian studies of return predictability allow for uncertainty in the parameters in (1), but assume flat priors (see Barberis (2000), Brandt, Goyal, Santa-Clara, and Stroud (2005), Johannes, Polson, and Stroud (2002), Skoulakis (2007) and Stambaugh (1999)). As Wachter (2010) shows, flat or nearly-flat priors imply a degree of predictability that is hard to justify economically. Other studies (Kandel and Stambaugh (1996), Pastor and Stambaugh (2009), Shanken and Tamayo (2011), Wachter and Warusawitharana (2009))

\footnotetext{
${ }^{1}$ However, note that our investor is Bayesian, rather than ambiguity averse (e.g. Chen and Epstein (2002)). Our priors are equivalent to placing a point mass on $\beta=0$ in (1).
} 
investigate the impact of economically informed prior beliefs. These studies nonetheless assume that the investor places a probability of one on the predictability of returns. However, an investor who thinks that (2) represents a compelling null hypothesis will have a prior that places some weight on the possibility that returns are not predictable at all.

Our work also relates to the Bayesian model selection methods of Avramov (2002) and Cremers (2002). In these studies, the investor has a prior probability over the full set of possible linear models that make use of a given set of predictor variables. Thus the setting of these papers is more complex than ours in that many predictor variables are considered. However, these papers also make the assumption that the predictor variables are either nonstochastic, or that their shocks are uncorrelated with shocks to returns. These assumptions are frequently satisfied in a standard ordinary least squares regression, but rarely satisfied in a predictive regression. In contrast, we are able to formulate and solve the Bayesian investor's problem when the regressor is stochastic and correlated with returns.

When we apply our methods to the dividend-price ratio, we find that an investor who believes that there is a $50 \%$ probability of predictability prior to seeing the data updates to a $86 \%$ posterior probability after viewing quarterly postwar data. We find average certainty equivalent returns of $1 \%$ per year for an investor whose prior probability in favor of predictability is just 20\%. For an investor who believes that there is a 50/50 chance of return predictability, certainty equivalent returns are $1.72 \%$.

We also empirically evaluate the effect of correctly incorporating the initial observation of the dividend-price ratio into the likelihood (the exact likelihood approach) versus the more common conditional likelihood approach. In the conditional likelihood approach, the initial observation of the predictor variable is treated as a known parameter rather than as a draw from the data generating process. We find that the the unconditional risk premium is poorly estimated when we condition on the first observation. However, when this is treated as a draw from the data generating process, the expected return is estimated reliably. Surprisingly, the posterior mean of the unconditional risk premium is notably lower than the sample average.

Finally, when we examine the evolution of posterior beliefs over the postwar period, we 
find substantial differences between the beliefs implied by our approach, which treats the regressor as stochastic and realistically captures the relation between the regressor and returns, and beliefs implied by assuming non-stochastic regressors. In particular, our approach implies that the belief in the predictability of returns rises dramatically over the 2000-2005 period while approaches assuming fixed regressors imply a decline. We also evaluate out-ofsample performance over this period, and show that our method leads to superior performance both when compared with a strategy based on sample averages, and when compared with a strategy implied by OLS regression.

The remainder of the paper is organized as follows. Section 2 describes our statistical method and contrasts it with alternative approaches. Section 3 describes our empirical results. Section 4 concludes.

\section{Statistical Method}

\subsection{Data generating processes}

Let $r_{t+1}$ denote continuously compounded excess returns on a stock index from time $t$ to $t+1$ and $x_{t}$ the value of a (scalar) predictor variable. We assume that this predictor variable follows the process

$$
x_{t+1}=\theta+\rho x_{t}+v_{t+1} .
$$

Stock returns can be predictable, in which case they follow the process (1) or unpredictable, in which case they follow the process $(2){ }^{2}$ In either case, errors are serially uncorrelated,

\footnotetext{
${ }^{2}$ The model we adopt for stock return predictability is assumed by Kandel and Stambaugh (1996), Campbell and Viceira (1999), Stambaugh (1999), Barberis (2000) and many subsequent studies. The idea that the price-dividend ratio can predict returns is motivated by present-value models of prices (see Campbell and Shiller (1988)). We have examined the possibility of adding lagged returns on the right hand side of both the return and predictor variable regression; however the coefficients are insignificant.
} 
homoskedastic, and jointly normal:

$$
\left[\begin{array}{c}
u_{t+1} \\
v_{t+1}
\end{array}\right] \mid r_{t}, \ldots, r_{1}, x_{t}, \ldots, x_{0} \sim N(0, \Sigma),
$$

and

$$
\Sigma=\left[\begin{array}{cc}
\sigma_{u}^{2} & \sigma_{u v} \\
\sigma_{u v} & \sigma_{v}^{2}
\end{array}\right]
$$

As we show below, the correlation between innovations to returns and innovations to the predictor variable implies that (3) affects inference about returns, even when there is no predictability.

When the process (3) is stationary, i.e. $\rho$ is between -1 and 1 , the predictor variable has an unconditional mean of

$$
\mu_{x}=\frac{\theta}{1-\rho}
$$

and a variance of

$$
\sigma_{x}^{2}=\frac{\sigma_{v}^{2}}{1-\rho^{2}}
$$

These follow from taking unconditional means and variances on either side of (3). Note that these are population values conditional on knowing the parameters. Given these, the population $R^{2}$ is defined as

$$
\text { Population } R^{2}=\frac{\beta^{2} \sigma_{x}^{2}}{\beta^{2} \sigma_{x}^{2}+\sigma_{u}^{2}} \text {. }
$$

\subsection{Prior Beliefs}

The investor faces uncertainty both about the model (i.e. whether returns are predictable or not), and about the parameters of the model. We represent this uncertainty through a hierarchical prior. There is a probability $q$ that investors face the distribution given by (1), (3) and (4). We denote this state of the world $H_{1}$. There is a probability $1-q$ that investors face the distribution given by (2), (3) and (4). We denote this state of the world $H_{0}$. As we will show, the stochastic properties of $x$ have relevance in both cases. 
The prior information on the parameters is conditional on $H_{i}$. Let

$$
b_{0}=[\alpha, \theta, \rho]^{\top}
$$

and

$$
b_{1}=[\alpha, \beta, \theta, \rho]^{\top}
$$

Note that $p\left(b_{1}, \Sigma \mid H_{1}\right)$ can also be written as $p\left(\beta, b_{0}, \Sigma \mid H_{1}\right) .{ }^{3}$ We set the prior on $b_{0}$ and $\Sigma$ so that

$$
p\left(b_{0}, \Sigma \mid H_{0}\right)=p\left(b_{0}, \Sigma \mid H_{1}\right)=p\left(b_{0}, \Sigma\right) .
$$

We assume the investor has uninformative beliefs on these parameters. We follow the approach of Stambaugh (1999) and Zellner (1996), and derive a limiting Jeffreys prior as explained in Appendix A. As Appendix A shows, this limiting prior takes the form

$$
p\left(b_{0}, \Sigma\right) \propto\left\{\begin{array}{cl}
\sigma_{x} \sigma_{u}|\Sigma|^{-\frac{5}{2}} & \rho \in(-1,1) \\
0 & \text { otherwise }
\end{array}\right.
$$

Equation 8 implies that the process for $x_{t}$ is stationary and that the mean (6) and variance (7) are well defined. Stationarity of $x_{t}$ is a standard assumption in the return predictability literature. Studies that rely on ordinary least squares make this assumption at least implicitly, since without it standard asymptotic arguments fail. Other recent studies (e.g. Cochrane (2008), Van Binsbergen and Koijen (2010)) explicitly assume stationarity. In Section 3.6, we discuss how this assumption affects our results.

The parameter that distinguishes $H_{0}$ from $H_{1}$ is $\beta$. One approach would be to write down a prior distribution for $\beta$ unconditional on the remaining parameters. However, there are advantages to forming priors on $\beta$ jointly with priors on other parameters. For example, a high variance of $x_{t}$ might lower one's prior on $\beta$, while a large residual variance of $r_{t}$ might raise it. Rather than placing a prior on $\beta$ directly, we follow Wachter and Warusawitharana (2009) and place a prior on the population $R^{2}$. To implement this prior on the $R^{2}$, we place

\footnotetext{
${ }^{3}$ Formally we could write down $p\left(b_{1}, \Sigma \mid H_{0}\right)$ by assuming $p\left(\beta \mid b_{0}, \Sigma, H_{0}\right)$ is a point mass at zero.
} 
a prior on "normalized" $\beta$, that is $\beta$ adjusted for the variance of $x$ and the variance of $u$. Let

$$
\eta=\sigma_{u}^{-1} \sigma_{x} \beta
$$

denote normalized $\beta$. We assume that prior beliefs on $\eta$ are given by

$$
\eta \mid H_{1} \sim N\left(0, \sigma_{\eta}^{2}\right)
$$

The population $R^{2}$ is closely related to $\eta$ :

$$
\text { Population } R^{2}=\frac{\beta^{2} \sigma_{x}^{2}}{\beta^{2} \sigma_{x}^{2}+\sigma_{u}^{2}}=\frac{\eta^{2}}{\eta^{2}+1} .
$$

Equation (10) provides a mapping between a prior distribution on $\eta$ and a prior distribution on the population $R^{2}$. Given an $\eta$ draw, an $R^{2}$ draw can be computed using (10).

A prior on $\eta$ implies a hierarchical prior on $\beta$. The prior for $\eta,(9)$, implies

$$
\beta \mid \alpha, \theta, \rho, \Sigma \sim N\left(0, \sigma_{\beta}^{2}\right)
$$

where

$$
\sigma_{\beta}=\sigma_{\eta} \sigma_{x}^{-1} \sigma_{u}
$$

Because $\sigma_{x}$ is a function of $\rho$ and $\sigma_{v}$, the prior on $\beta$ is also implicitly a function of these parameters. The parameter $\sigma_{\eta}$ indexes the degree to which the prior is informative. As $\sigma_{\eta} \rightarrow \infty$, the prior over $\beta$ becomes uninformative; all values of $\beta$ are viewed as equally likely. As $\sigma_{\eta} \rightarrow 0$, the prior converges to $p\left(b_{0}, \Sigma\right)$ multiplied by a point mass at 0 , implying a dogmatic view in no predictability. Combining (11) with (8) implies the joint prior under $H_{1}$ :

$$
\begin{aligned}
p\left(b_{1}, \Sigma \mid H_{1}\right) & =p\left(\beta \mid b_{0}, \Sigma, H_{1}\right) p\left(b_{0} \mid H_{1}\right) \\
& \propto \frac{1}{\sqrt{2 \pi \sigma_{\eta}^{2}}} \sigma_{x}^{2}|\Sigma|^{-\frac{5}{2}} \exp \left\{-\frac{1}{2} \beta^{2}\left(\sigma_{\eta}^{2} \sigma_{x}^{-2} \sigma_{u}^{2}\right)^{-1}\right\} .
\end{aligned}
$$

Jeffreys invariance theory provides an independent justification for modeling priors on $\beta$ as (11). Stambaugh (1999) shows that the limiting Jeffreys prior for $b_{1}$ and $\Sigma$ equals

$$
p\left(b_{1}, \Sigma \mid H_{1}\right) \propto \sigma_{x}^{2}|\Sigma|^{-\frac{5}{2}} .
$$


This prior corresponds to the limit of (12) as $\sigma_{\eta}$ approaches infinity. Modeling the prior for $\beta$ as depending on $\sigma_{x}$ not only has a convenient interpretation in terms of the distribution of the $R^{2}$, but also implies that an infinite prior variance represents ignorance as defined by Jeffreys (1961). Note that a prior on $\beta$ that is independent of $\sigma_{x}$ would not have this property.

Figure 1 shows the resulting distribution for the population $R^{2}$ for various values of $\sigma_{\eta}$. Panel A shows the distribution conditional on $H_{1}$ while Panel B shows the unconditional distribution. More precisely, for any value $k$, Panel A shows the prior probability that the $R^{2}$ exceeds $k$, conditional on the existence of predictability. For large values of $\sigma_{\eta}$, e.g. 100, the prior probability that the $R^{2}$ exceeds $k$ across the relevant range of values for the $R^{2}$ is close to one. The lower the value of $\sigma_{\eta}$, the less variability in $\beta$ around its mean of zero, and the lower the probability that the $R^{2}$ exceeds $k$ for any value of $k$. Panel $\mathrm{B}$ shows the unconditional probability that the $R^{2}$ exceeds $k$ for any value of $k$, assuming that the prior probability of predictability, $q$, is equal to 0.5 . By the definition of conditional probability:

$$
p\left(R^{2}>k\right)=p\left(R^{2}>k \mid H_{1}\right) q
$$

Therefore Panel B takes the values in Panel A and scales them down by 0.5.

\subsection{Likelihood}

\subsubsection{Likelihood under $H_{1}$}

Under $H_{1}$, returns and the predictor variable follow the joint process given in (1) and (3). It is convenient to group observations on returns and contemporaneous observations on the state variable into a matrix $Y$ and lagged observations on the state variable and the constant into a matrix $X$. Let

$$
Y=\left[\begin{array}{cc}
r_{1} & x_{1} \\
\vdots & \vdots \\
r_{T} & x_{T}
\end{array}\right] \quad X=\left[\begin{array}{cc}
1 & x_{0} \\
\vdots & \vdots \\
1 & x_{T-1}
\end{array}\right]
$$


and let

$$
\begin{aligned}
z & =\operatorname{vec}(Y) \\
Z_{1} & =I_{2} \otimes X .
\end{aligned}
$$

In the above, the vec operator stacks the elements of the matrix columnwise. It follows that the likelihood conditional on $H_{1}$ and on the first observation $x_{0}$ takes the form of

$$
p\left(D \mid b_{1}, \Sigma, x_{0}, H_{1}\right)=|2 \pi \Sigma|^{-\frac{T}{2}} \exp \left\{-\frac{1}{2}\left(z-Z_{1} b_{1}\right)^{\top}\left(\Sigma^{-1} \otimes I_{T}\right)\left(z-Z_{1} b_{1}\right)\right\}
$$

(see Zellner (1996)).

The likelihood function (14) conditions on the first observation of the predictor variable, $x_{0}$. Stambaugh (1999) argues for treating $x_{0}$ and $x_{1}, \ldots, x_{T}$ symmetrically: as random draws from the data generating process. If the process for $x_{t}$ is stationary and has run for a substantial period of time, then results in Hamilton (1994, p. 265) imply that $x_{0}$ is

a draw from a normal distribution with mean $\mu_{x}$ and standard deviation $\sigma_{x}$. Combining the likelihood of the first observation with the likelihood of the remaining $T$ observations produces

$$
\begin{aligned}
p\left(D \mid b_{1}, \Sigma, H_{1}\right)=\left|2 \pi \sigma_{x}^{2}\right|^{-\frac{1}{2}}|2 \pi \Sigma|^{-\frac{T}{2}} \exp \{ & -\frac{1}{2}\left(x_{0}-\mu_{x}\right)^{2} \sigma_{x}^{-2} \\
& \left.-\frac{1}{2}\left(z-Z_{1} b_{1}\right)^{\top}\left(\Sigma^{-1} \otimes I_{T}\right)\left(z-Z_{1} b_{1}\right)\right\} .
\end{aligned}
$$

Following Box and Tiao (1973), we refer to (14) as the conditional likelihood and (15) as the exact likelihood.

\subsubsection{Likelihood under $H_{0}$}

Under $H_{0}$, returns and the predictor variable follow the processes given in (2) and (3). Let

$$
Z_{0}=\left[\begin{array}{cc}
\iota_{T} & 0_{T \times 2} \\
0_{T \times 1} & X
\end{array}\right],
$$

where $\iota_{T}$ is the $T \times 1$ vector of ones. Then the conditional likelihood can be written as

$$
p\left(D \mid b_{0}, \Sigma, x_{0}, H_{0}\right)=|2 \pi \Sigma|^{-\frac{T}{2}} \exp \left\{-\frac{1}{2}\left(z-Z_{0} b_{0}\right)^{\top}\left(\Sigma^{-1} \otimes I_{T}\right)\left(z-Z_{0} b_{0}\right)\right\} .
$$


The conditional likelihood takes the same form as in the seemingly unrelated regression model (see Ando and Zellner (2010)). Using similar reasoning as in the $H_{1}$ case, the exact likelihood is given by

$$
\begin{aligned}
p\left(D \mid b_{0}, \Sigma, H_{0}\right)=\left|2 \pi \sigma_{x}^{2}\right|^{-\frac{1}{2}}|2 \pi \Sigma|^{-\frac{T}{2}} \exp \{- & \frac{1}{2}\left(x_{0}-\mu_{x}\right)^{2} \sigma_{x}^{-2} \\
& \left.-\frac{1}{2}\left(z-Z_{0} b_{0}\right)^{\top}\left(\Sigma^{-1} \otimes I_{T}\right)\left(z-Z_{0} b_{0}\right)\right\} .
\end{aligned}
$$

As above, we refer to (16) as the conditional likelihood and (17) as the exact likelihood.

\subsection{Posterior distribution}

The investor updates his prior beliefs to form the posterior distribution upon seeing the data. As we discuss below, this posterior requires the computation of two quantities: the posterior of the parameters conditional on the absence or presence of return predictability, and the posterior probability that returns are predictable. Given these two quantities, we can simulate from the posterior distribution.

To compute the posteriors, we apply Bayes' rule conditional on the model:

$$
p\left(b_{i}, \Sigma \mid H_{i}, D\right) \propto p\left(D \mid b_{i}, \Sigma, H_{i}\right) p\left(b_{i}, \Sigma \mid H_{i}\right), \quad i=0,1 .
$$

Because $\sigma_{x}$ is a nonlinear function of the underlying parameters, the posterior distributions conditional on $H_{0}$ and $H_{1}$ are nonstandard and must by computed numerically. We can sam-

ple from these distributions quickly and accurately using the Metropolis-Hastings algorithm (see Chib and Greenberg (1995), Johannes and Polson (2006)). See Appendix B for details.

Let $\bar{q}$ denote the posterior probability that excess returns are predictable. By definition,

$$
\bar{q}=p\left(H_{1} \mid D\right)
$$

It follows from Bayes' rule, that

$$
\bar{q}=\frac{\mathcal{B}_{10} q}{\mathcal{B}_{10} q+(1-q)}
$$


where

$$
\mathcal{B}_{10}=\frac{p\left(D \mid H_{1}\right)}{p\left(D \mid H_{0}\right)}
$$

is the Bayes factor for the alternative hypothesis of predictability against the null of no predictability. The Bayes factor is a likelihood ratio in that it is the likelihood of return predictability divided by the likelihood of no predictability. However, it differs from the standard likelihood ratio in that the likelihoods $p\left(D \mid H_{i}\right)$ are not conditional on the values of the parameters. These likelihoods are given by

$$
p\left(D \mid H_{i}\right)=\int p\left(D \mid b_{i}, \Sigma, H_{i}\right) p\left(b_{i}, \Sigma \mid H_{i}\right) d b_{i} d \Sigma, \quad i=0,1
$$

To form these likelihoods, the likelihoods conditional on parameters (the likelihood functions generally used in classical statistics) are integrated over the prior distribution of the parameters. Under our distributions, these integrals cannot be computed analytically. However, the Bayes factor (20) can be computed directly using the generalized Savage-Dickey density ratio (Dickey (1971), Verdinelli and Wasserman (1995)). Details can be found in Appendix C.

Putting these two pieces together, we draw from the posterior parameter distribution by drawing from $p\left(b_{1}, \Sigma \mid D, H_{1}\right)$ with probability $\bar{q}$ and from $p\left(b_{0}, \Sigma \mid D, H_{0}\right)$ with probability $1-\bar{q}$

\subsection{The exogenous regressor approach}

Our likelihood and prior involves not only the process for returns conditional on the lagged predictor, but the process for the predictor variable itself. A common alternative is to form a likelihood function from the return equation only. That is, the likelihood function is taken to be:

$$
p\left(R \mid X, \alpha, \beta, \sigma_{u}, H_{1}\right)=\left(2 \pi \sigma_{u}^{2}\right)^{-\frac{T}{2}} \exp \left\{-\frac{1}{2} \sum_{t=0}^{T-1}\left(r_{t+1}-\alpha-\beta x_{t}\right)^{2} \sigma_{u}^{-2}\right\},
$$

for $R=\left[r_{1}, \ldots, r_{T}\right]^{\top}$. This is combined with a prior over $\alpha, \beta$ and $\sigma_{u}$ only.

This approach is appealingly simple, but is it valid? In fact (22) is not a valid likelihood function under reasonable conditions. The reason is that, unless $x_{t}$ is strictly exogenous, 
conditioning on the entire time series of $x_{t}$, as in (22), implies a different distribution for $r_{t+1}$ than conditioning on $x_{t}$ alone. Namely, conditional on the future values of $x, r_{t+1}$ is not normally distributed with mean $\alpha+\beta x_{t}$ and variance $\sigma_{u}$ :

$$
p\left(r_{t+1} \mid x_{t+1}, x_{t}, \alpha, \beta, \sigma_{u}, H_{1}\right) \neq p\left(r_{t+1} \mid x_{t}, \alpha, \beta, \sigma_{u}, H_{1}\right) .
$$

The value of $x_{t+1}$ conveys information about the shock $v_{t+1}$, which in turn conveys information about $u_{t+1}$ (because they are correlated), and $u_{t+1}$ conveys information about $r_{t+1}$.

Is there perhaps some other way to justify using the right hand side of (22) as a likelihood? The true (conditional) likelihood arises from taking the product of terms

$$
p\left(D \mid x_{0}, b_{1}, \Sigma, H_{1}\right)=\prod_{t=0}^{T-1} p\left(r_{t+1}, x_{t+1} \mid r_{t}, x_{t}, b_{1}, \Sigma, H_{1}\right){ }^{4}
$$

One could separate out the terms in the product as follows

$$
\prod_{t=0}^{T-1} p\left(r_{t+1} \mid x_{t}, \alpha, \beta, \sigma_{u}\right) p\left(x_{t+1} \mid r_{t+1}, x_{t}, b_{1}, \Sigma\right) .
$$

However, the second term in (23) depends on $\alpha, \beta$ and $\sigma_{u}$. It is not, therefore, a constant when one applies Bayes rule to inference about these parameters. Using the right hand side of (22) thus requires either incorrect conditioning on the time path of $x$, or an incorrect computation of the posterior.

At the root of the problem is the fact that the similarity between the likelihood in the linear regression model in the time series setting and under OLS is only apparent. In a time series setting, it is not valid to condition on the entire time path of the "independent" variable. The differences ultimately come down to the interpretation of the term $u_{t}$. In a standard OLS setting, $u_{t}$ is an error, and is thus uncorrelated with the independent variable at all leads and lags. In a time series setting, it is not an error, but rather a shock, and this independence does not hold. ${ }^{5}$

\footnotetext{
${ }^{4}$ Note this likelihood function still conditions on $x_{0}$, and so is the conditional rather than the exact likelihood.

${ }^{5}$ This point is also emphasized by Stambaugh (1999).
} 
Of course, there is a special case in which it is correct to condition on the time path of $x_{t}$. This is when the errors $u_{t}$ and $v_{t}$ are known to be uncorrelated at all leads and lags. In this case, $x_{t}$ is strictly exogenous. This is an unrealistic assumption in a time series setting, particularly for the dividend-price ratio (or other scaled measures of market value), because future returns are by definition likely to be correlated with past prices. Indeed, the correlation between $u_{t}$ and $v_{t}$ is close to -1 . While strict exogeneity could be enforced in the prior, it is clearly counterfactual. Fortunately it is not necessary: our analysis shows how inference can proceed without it. In what follows, we will compare our results to what would happen under this approach, which, for simplicity, we refer to as the non-stochastic regressor approach.

\section{Results}

\subsection{Data}

We use data from the Center for Research on Security Prices (CRSP). We compute excess stock returns by subtracting the continuously compounded 3-month Treasury bill return from the continuously compounded return on the value-weighted CRSP index at a quarterly frequency. Following a large empirical literature on return predictability, we focus on the dividend-price ratio as the regressor because the present-value relation between prices and returns suggests that it should capture variables that predict stock returns. The dividendprice ratio is computed by dividing the dividend payout over the previous 12 months with the current price of the stock index. The use of 12 months of data accounts for seasonalities in dividend payments. We use the logarithm of the dividend-price ratio as the predictor variable. Data are quarterly from 1952 to $2009 .{ }^{6}$

\footnotetext{
${ }^{6}$ We obtain very similar results at an annual and monthly frequency.
} 


\subsection{Bayes factors and posterior means}

Table 1 reports Bayes factors for various priors. Four values of $\sigma_{\eta}$ are considered: 0.051, 0.087, 0.148 and 100. These translate into values of $P\left(R^{2}>.01 \mid H_{1}\right)$ (the prior probability that the $R^{2}$ exceeds 0.01 ) equal to $0.05,0.25,0.50$ and 0.99 respectively. These $R^{2}$ s should be interpreted in terms of regressions performed at a quarterly frequency. Bayes factors are reported for the exact likelihood, and, to evaluate the importance of including the initial term, the conditional likelihood as well.

Table 1 shows that the Bayes factor is hump-shaped in $P\left(R^{2}>0.01 \mid H_{1}\right)$. For small values, the Bayes factor is close to one. For large values, the Bayes factor is close to zero. Both results can be understood using the formula for the Bayes factor in (20) and for the likelihoods $p\left(D \mid H_{i}\right)$ in (21). For low values of this probability, the investor imposes a very tight prior on the $R^{2}$. Therefore the hypotheses that returns are predictable and that returns are unpredictable are nearly the same. It follows from (21) that the likelihoods of the data under these two scenarios are nearly the same and that the Bayes factor is nearly one. This is intuitive: when two hypotheses are close, a great deal of data are required to distinguish one from the other.

The fact that the Bayes factor approaches zero as $P\left(R^{2}>.01 \mid H_{1}\right)$ continues to increase is less intuitive. The reduction in Bayes factors implies that, as the investor allows a greater range of values for the $R^{2}$, the posterior probability that returns are predictable approaches zero. This effect is known as Bartlett's paradox, and was first noted by Bartlett (1957) in the context of distinguishing between uniform distributions. As Kass and Raftery (1995) discuss, Bartlett's paradox makes it crucial to formulate an informative prior on the parameters that differ between $H_{0}$ and $H_{1}$. The mathematics leading to Bartlett's paradox are most easily seen in a case where Bayes factors can be computed in closed form. However, we can obtain an understanding of the paradox based on the form of the likelihoods $p\left(D \mid H_{1}\right)$ and $P\left(D \mid H_{0}\right)$. These likelihoods involve integrating out the parameters using the prior distribution. If the prior distribution on $\beta$ is highly uninformative, the prior places a large 
amount of mass in extreme regions of the parameter space. In these regions, the likelihood of the data conditional on the parameters will be quite small. At the same time, the prior places a relatively small amount of mass in the regions of the parameter space where the likelihood of the data is large. Therefore $P\left(D \mid H_{1}\right)$ (the integral of the likelihood under $H_{1}$ ) is small relative to $P\left(D \mid H_{0}\right)$ (the integral of the likelihood under $H_{0}$ ).

Table 1 also shows that there are substantial differences between the Bayes factors resulting from the exact versus the conditional likelihood. ${ }^{7}$ The Bayes factors resulting from the exact likelihood are larger than those resulting from the conditional likelihood, thus implying a greater posterior probability of return predictability. This difference reflects the fact that the posterior mean of $\beta$, conditional on $H_{1}$, is higher for the exact likelihood than for the conditional likelihood, and the posterior mean is $\rho$ is lower. ${ }^{8}$

\subsection{The long-run equity premium}

For the predictability model, the expected excess return on stocks (the equity premium) varies over time. In the long run, however, the current value of $x_{t}$ becomes irrelevant. Under our assumptions $x_{t}$ is stationary with mean $\mu_{x}$, and therefore $r_{t}$ is also stationary with mean

$$
\mu_{r}=E\left[\alpha+\beta x_{t}+u_{t+1} \mid b_{1}, \Sigma\right]=\alpha+\beta \mu_{x}
$$

As is the case with $\mu_{x}$, this is a population value that conditions on the value of the parameters. For the no-predictability model, $\mu_{r}$ is simply equal to $\alpha$. We can think of $\mu_{r}$ as the average equity premium; the fact that it is "too high" constitutes the equity premium

\footnotetext{
${ }^{7}$ We are not the first to note the importance of the first observation. See, for example, Poirier (1978).

${ }^{8}$ The source of this negative relation is the negative correlation between shocks to returns and shocks to the predictor variable. Suppose that a draw of $\beta$ is below its value predicted by ordinary least squares (OLS). This implies that the OLS value for $\beta$ is "too high", i.e. in the sample shocks to the predictor variable are followed by shocks to returns of the same sign. Therefore shocks to the predictor variable tend to be followed by shocks to the predictor variable that are of different signs. Thus the OLS value for $\rho$ is "too low". This explains why values of the posterior mean of $\rho$ are higher for low values of $P\left(R^{2}>0.01 \mid H_{1}\right)$ (and hence low values of the posterior mean of $\beta$ ) than for high values, and higher than the ordinary least squares estimate.
} 
puzzle (Mehra and Prescott (1985)), and it is often computed by simply taking the sample average of excess returns.

The posterior expectation of $\mu_{r}$ under various specifications is shown in the fifth column of Table 1. Because differences in the expected return arise from differences in the posterior mean of the predictor variable $x$, the table also reports the posterior mean of $\mu_{x}$. The differences in the long-run equity premium are striking. The sample average of the (continuously compounded) excess return on stocks over this period is $4.49 \%$. However, assuming the exact likelihood implies produces a range for this excess return between $3.45 \%$ and $3.90 \%$ depending on the strength of the prior. Why is the equity premium in these cases as much as a full percentage point lower?

To answer this question, it is helpful to look at the posterior means of the predictor variable, reported in the next column of Table 1. For the exact likelihood specification, the posterior mean of the log dividend yield ranges from -3.25 to -3.40 . The sample mean is -3.54 . It follows that the shocks $v_{t}$ over the sample period must be negative on average. Because of the negative correlation between shocks to the dividend price ratio and to expected returns, the shocks $u_{t}$ must be positive on average. Therefore the posterior mean lies below the sample mean.

Continuing with the exact likelihood case, the posterior mean of $\mu_{x}$ is highest (and hence furthest from the sample mean) in the no-predictability model, and becomes lower as the prior becomes less dogmatic. Excess returns follow this pattern in reverse, namely they are lowest (and furthest from the sample mean) for the no-predictability model and highest for the predictability model with the least dogmatic prior. This effect may arise from the persistence $\rho$. The more dogmatic the prior, the closer the posterior mean of the persistence is to one. The more persistent the process, the more likely the positive shocks are to accumulate, and the more the sample mean is likely to deviate from the true posterior mean.

The results are very different when the conditional likelihood is used, as shown in Panel B. For the no-predictability model, $\mu_{r}=\alpha$ is equal to the sample mean. However, as long as 
there is some predictability, estimation of $\mu_{r}$ depends on $\mu_{x}$, which is unstable due to the presence of $1-\rho$ in the denominator. It is striking that, in contrast to our main specification, the conditional likelihood specification has great difficulty in pinning down the mean of expected excess stock returns.

\subsection{The posterior distribution}

We now examine the posterior probability that excess returns are predictable. For convenience, we present results for our main specification that uses the exact likelihood. As a first step, we examine the posterior distribution for the $R^{2}$.

\section{The posterior distribution of the $R^{2}$}

Figure 2 displays the prior and posterior distribution of the $R^{2}$. For now we assume that prior beliefs are given by $P\left(R^{2}>1 \% \mid H_{1}\right)=0.50$ and $q=0.5$; below we examine robustness to changes in these values. Panel A shows $P\left(R^{2}>k\right)$ as a function of $k$ for both the prior and the posterior; this corresponds to 1 minus the cumulative density function of the $R^{2}{ }^{9}$ Panel A demonstrates a rightward shift for the posterior for values of $k$ below (roughly) $2 \%$. While the prior implies $P\left(R^{2}>1 \%\right)=0.25$, the posterior implies $P\left(R^{2}>1 \%\right)$ close to 0.50 . Thus, after observing the data, an investor revises his beliefs on the existence and strength of predictability substantially upward.

Panel $\mathrm{B}$ shows the probability density function of the $R^{2}$. The prior places the highest density on low values of the $R^{2}$. The posterior however places high density in the region around $2 \%$ and has lower density than the prior for $R^{2}$ values close to zero. The evidence in favor of predictability, with a moderate $R^{2}$, is sufficient to overcome the investor's initial skepticism.

\footnotetext{
${ }^{9}$ This figures shows the unconditional posterior probability that the $R^{2}$ exceeds $k$; that is, they do not condition on the existence of predictability.
} 


\section{The posterior probability of return predictability}

Table 2 shows how various statistics on the posterior distribution vary as the prior distribution changes. Panel A presents the posterior probabilities of predictability as a function of the investor's prior about the existence of predictability, $q$, and the prior belief on the strength of predictability. The posterior probability is increasing in $q$ and hump-shaped in the strength of the prior, reflecting the fact that the Bayes factors are hump-shaped in the strength of the prior. An investor with moderate beliefs about the probability that returns are predictable revises these beliefs sharply upward. For example, an investor with $q=0.5$ and $P\left(R^{2}>.01 \mid H_{1}\right)=0.50$ concludes that the posterior likelihood of predictability equals 0.86. This result is robust to a wide range of choices for $P\left(R^{2}>.01 \mid H_{1}\right)$. As the table shows, $P\left(R^{2}>.01 \mid H_{1}\right)=0.25$ implies a posterior probability of 0.87 . The posterior probability falls off dramatically for $P\left(R^{2}>.01 \mid H_{1}\right)=0.99$; for these very diffuse priors (which imply what might be considered an economically unreasonable amount of predictability), the Bayes factors are close to zero. ${ }^{10}$ Panels $\mathrm{B}$ and $\mathrm{C}$ show reasonably high means of the $\beta$ and the $R^{2}$, except for the most diffuse prior.

The above analysis evaluates the statistical evidence on predictability. The Bayesian approach also enables us to study the economic gains from market timing. In particular, we can evaluate the certainty equivalent loss from failing to time the market under different priors on the existence and strength of predictability.

\section{Certainty equivalent returns}

We now measure the economic significance of the predictability evidence using certainty equivalent returns. We assume an investor who maximizes

$$
E\left[\frac{W_{T+1}^{1-\gamma}}{1-\gamma} \mid D\right]
$$

\footnotetext{
${ }^{10}$ See this discussion in Section 3.2 on Bartlett's paradox.
} 
for $\gamma=5$, where $W_{T+1}=W_{T}\left(w e^{r_{T+1}+r_{f, T}}+(1-w) e^{r_{f, T}}\right)$, and $w$ is the weight on the risky asset. The expectation is taken with respect to the predictive distribution

$$
p\left(r_{T+1} \mid D\right)=\bar{q} p\left(r_{T+1} \mid D, H_{1}\right)+(1-\bar{q}) p\left(r_{T+1} \mid D, H_{0}\right)
$$

where

$$
p\left(r_{T+1} \mid D, H_{i}\right)=\int p\left(r_{T+1} \mid x_{T}, b_{i}, \Sigma, H_{i}\right) p\left(b_{i}, \Sigma \mid D, H_{i}\right) d b_{i} d \Sigma
$$

for $i=0,1$. A draw $r_{T+1}$ from the distribution $p\left(r_{T+1} \mid x_{T}, b_{1}, \Sigma\right)$ is given by (1) with probability $\bar{q}$ and (2) with probability $1-\bar{q}$.

For any portfolio weight $w$, we can compute the certainty equivalent return (CER) as solving

$$
\frac{\exp \{(1-\gamma) \mathrm{CER}\}}{1-\gamma}=E\left[\frac{\left(w e^{r_{T+1}+r_{f, T}}+(1-w) e^{r_{f, T}}\right)^{1-\gamma}}{1-\gamma} \mid D\right]
$$

Following Kandel and Stambaugh (1996), we measure utility loss as the difference between certainty equivalent returns from following the optimal strategy and from following a suboptimal strategy. We define the sub-optimal strategy as the strategy that the investor would follow if he believes that there is no predictability. Note, however, that the expectation in (24) is computed with respect to the same distribution for both the optimal and sub-optimal strategy.

Panel D of Table 2 shows the difference in certainty equivalent returns as described above. These differences are averaged over the posterior distribution for $x$ to create a difference that is not conditional on any specific value. The data indicate economically meaningful economic losses from failing to time the market. Panel D shows that, for example, an investor with a prior on $\beta$ such that $P\left(R^{2}>.01 \mid H_{1}\right)=0.50$ and a $50 \%$ prior belief in the existence of return predictability would suffer a certainty equivalent loss of $1.72 \%$ (in annual terms) from failing to time the market. Higher values of $q$ imply greater certainty equivalent losses. 


\subsection{Evolution of the posterior distribution over time}

We next describe the evolution of the posterior distribution over time. This distribution exhibits surprising behavior over the 2000-2005 period. This behavior is a direct result of the stochastic properties of the predictor variable $x_{t}$. Unless stated otherwise, the results in this section are for the benchmark specification, namely, the priors given in Section 2.2 combined with the exact likelihood. The prior probability that the $R^{2}$ exceeds $1 \%$ and the prior probability of predictability are assumed to be 0.5 .

Starting in 1972, we compute the posterior distribution conditional on having observed data up to and including that year. We start in 1972 because this allows for twenty years of data for the first observation. The posterior is computed by simulating 500,000 draws and dropping the first 100,000. To save on computation time, we update the posterior every year. For reference, Figure 3 shows the time series of the log dividend-price ratio. As we will see, much of the behavior of the posterior distribution can be understood based on the time series of this ratio.

Figure 4 shows the posterior probability of predictability $(\bar{q})$ in Panel A (assuming a prior probability of 0.5 ). The solid line corresponds to our benchmark specification. This line is above $90 \%$ for most of the sample (it is actually at its lowest value at the end of the sample). In the 2000-2005 period, the probability is not distinguishable from one. This is surprising: intuition would suggest that the period in which the dividend-price ratio was falling far below its long-run mean (and during which returns were high regardless) would correspond to an exceptionally low posterior probability of predictability, not a high one. Indeed, it is surprising that data could ever lead the investor to a nearly $100 \%$ certainty about the predictability model.

Panel B, which shows log Bayes factors, gives another perspective on this result. Between 2000 and 2005, the Bayes factor in favor of predictability rises to values that dwarf any others

during the sample. The posterior probability takes these Bayes factors and maps them to the $[0,1]$ interval, so values as high as those shown in the figure are translated to posterior 
probabilities extremely close to one. Why is it that the Bayes factors rise so high?

An answer is suggested by the time series behavior of $\beta$ and $\rho$, shown in Figure 5 . The solid lines show the posterior distributions of $\beta$ and $\rho .{ }^{11}$ The dashed line shows OLS estimates. The posterior for $\beta$ lies below the OLS estimate for most of the period, while the posterior for $\rho$ lies above the OLS estimator for most of the period. An exception occurs in 2001, when the positions reverse. The posterior for $\beta$ lies above the OLS estimate and the posterior for $\rho$ lies below it. Note that the OLS estimate of $\beta$ is biased upwards and the OLS estimate of $\rho$ is biased downwards, so this switch is especially surprising.

The fact that the posterior $\rho$ rises to meet the OLS $\rho$, and even exceeds it, indicates that the model interprets the rise of the dividend-price ratio as occurring because of an unusual sequence of negative shocks $v_{t}$. Namely, negative shocks are more likely to occur after negative shocks during this period. This implies that positive shocks to $u_{t}$ are also more likely to follow negative shocks $v_{t}$ than they would in population, so OLS will in fact underestimate the true $\beta$ (or it will overestimate the true $\beta$ by less than usual).

This result is similar in spirit to that found in the frequentist analysis of Lewellen (2004) and Campbell and Yogo (2006) (see also the discussion in the survey, Campbell (2008)). It is also an example of how information about shocks that are correlated with errors from a forecasting model can help improve forecasts, as in Faust and Wright (2011). Figure 4 shows that the consequences of this result for model selection are quite large. This is because the no-predictability model implies, of course, that $\beta$ is zero. However, given that OLS finds a positive $\beta$, for the no-predictability model to be true, it must be the case that negative shocks to the dividend-price ratio were follows by negative shocks to returns. This is extremely unlikely, given the time series evidence and a stationary predictor variable. Thus the evidence comes to strongly favor the predictability model.

\footnotetext{
${ }^{11}$ For the argument below, it makes the most sense, strictly speaking, to examine the posterior distribution of $\beta$ conditional on the predictability model. However, because the posterior probability of this model is so close to one, this conditional posterior $\beta$ is nearly indistinguishable from the unconditional posterior $\beta$. The same is true for posterior $\rho$. Therefore, for simplicity, we focus on the unconditional posterior.
} 


\section{Comparison with the non-stochastic regressor approach}

This chain of inference requires knowledge of the behavior of shocks to the predictor variable. The non-stochastic regressor approach described in Section 2.5 eliminates such knowledge and leads to completely different inference over this time period. To fix ideas, we implement this approach using the standard assumption of a conjugate prior distribution. However, our findings do not depend on this assumption, as we discuss in Section 3.6.

We assume the following prior distribution on the return parameters:

$$
\begin{aligned}
{[\alpha, \beta]^{\top} \mid \sigma_{u}^{2}, H_{1} } & \sim N\left(0, g^{-1} \sigma_{u}^{2}\left(X^{\top} X\right)^{-1}\right) \\
\sigma_{u}^{2} \mid H_{1} & \sim I W\left(N_{0}-2, s_{0}\right)
\end{aligned}
$$

where $I W$ denotes the inverse Wishart distribution, and $g^{-1}, N_{0}$ and $s_{0}$ are parameters of the prior distribution. ${ }^{12}$ Note that prior for $\beta$ conditional on $\sigma_{u}$ is

$$
\beta \mid \sigma_{u}^{2}, H_{1} \sim N\left(0, g^{-1} \sigma_{u}^{2} T \hat{\sigma}_{x}^{-2}\right)
$$

where $\hat{\sigma}_{x}^{2}$ denotes the sample variance of $x$ :

$$
\hat{\sigma}_{x}^{2}=\frac{1}{T} \sum_{t=0}^{T-1}\left(x_{t}-\frac{1}{T} \sum_{s=0}^{T-1} x_{s}\right)^{2} .
$$

This allows us to construct these priors so that they are of comparable informativeness to our benchmark priors in Section 2.2 by setting

$$
g^{-1} T=\sigma_{\eta}^{2}
$$

The prior in (25) and (26) is equivalent to the $g$-prior of Zellner (1986), and is similar to specifications employed by Fernandez, Ley, and Steel (2001), Chipman, George, and McCulloch (2001), Avramov (2002), Cremers (2002), Wright (2008) and Stock and Watson

\footnotetext{
${ }^{12}$ In fact, because it is scalar the distribution of $\sigma_{u}^{2}$ is an inverse Gamma. We express it as a inverse Wishart for comparability to multivariate results later in the manuscript. We set $N_{0}$ equal to 40 and $s_{0}$ equal to the sum of squared errors over the sample, multiplied by $N_{0} / T$. The results are not sensitive to these choices. See Appendix E for further interpretation of these prior beliefs.
} 
(2012). As explained in Section 2.5, the likelihood function in the non-stochastic regressor case is given by (22).

In our time-series setting, (25) relies on incorrect conditioning: the investor must have foreknowledge of the entire time path of the predictor variable. Thus, the approach described here builds in the assumption of a non-stochastic regressor in two ways. First, the terms involving the predictor variable do not appear in the likelihood function. Second, it conditions on the entire time path of $x_{t}$ in forming the prior distribution. ${ }^{13}$

Appendix E describes the computation of Bayes factors and posterior probabilities in this case. The dashed line in Figure 4 shows the posterior probabilities and Bayes factors. Notably, the non-stochastic case does not exhibit the large upward spike in Bayes factors, nor do the posterior probabilities approach one in the 2000-2005 period. Rather, the posterior probabilities decline substantially in 1998-2000, and while they increase again after this, they remain a level lower than the earlier part of the sample. This behavior stems from the behavior of the OLS predictive coefficients (Figure 5), which follow a similar pattern. The benchmark case in Figure 4 combines this information with additional information contained in the shocks $v_{t}$, and therefore in $u_{t} .{ }^{14}$ As explained in the paragraphs above, this information makes it very unlikely that the no-predictability model holds over the 2000-2005 period. ${ }^{15}$

\footnotetext{
${ }^{13}$ An alternative approach would be to form a conjugate $g$-prior over a multivariate system that includes the equation for the state variable. Under this approach, terms involving the predictor variable would appear in the prior and likelihood function. However, it would still involve incorrect conditioning in that the entire path of $x_{t}$ would be used in forming the prior. This approach is described in detail in Appendix D. Comparing the resulting posterior distribution with that from the one-equation conjugate prior case reveals that they differ up to a degrees of freedom adjustment arising from the need to estimate the correlation between the two equations.

${ }^{14}$ For the information in $v_{t}$ to matter, there must be a non-zero correlation between $u$ and $v$. As Appendix G shows, in the case of the yield spread, the benchmark and non-stochastic cases look nearly identical in part because the correlation between shocks to the yield spread and shocks to returns is low in magnitude.

${ }^{15}$ The effect is most dramatic over the 2000-2005 period, but holds to some extent in other parts of the sample period as well. This is one of the reasons why Bayes factors for the benchmark case lie above those for the non-stochastic case throughout the sample.
} 


\subsection{The role of the prior and likelihood in determining Bayes fac- tors}

As Section 3.5 shows, whether one models the predictor variable as stochastic or not has a large impact on inference. This section delves more deeply into the reasons for this difference.

Clearly there are many differences between the stochastic (benchmark) and non-stochastic case. Most fundamentally, the benchmark case requires specifying a likelihood function for the data on the predictor variable. This in turn requires a prior over the parameters of this likelihood function. By modeling the predictor as non-stochastic, one appears to avoid this step.

In specifying this prior, we assume that the predictor variable is stationary. Without this assumption, we could not define a prior over the $R^{2}$ (because the variance is not well-defined) nor would we have an exact likelihood function (there would be no well-defined distribution for $x_{0}$ ). As we discuss in Section 2.2, this assumption is standard in the return predictability literature, though it is not always stated explicitly. Thus in our setting stationarity is a natural assumption. Here, we seek to understand how it affects our results and why.

We first ask whether it matters if we use the exact or the conditional likelihood. We do this by comparing our benchmark case with one in which we use the conditional likelihood and keep all else the same. This is shown in Panel A of Figure 6. Using the conditional likelihood leads to lower Bayes factors, though the Bayes factors still spike up over the 2000-2005 period. The information from the first observation shifts the distribution of $\rho$ toward lower values because the mean of the predictor variable is sufficiently close to the first observation that a high variance of the predictor variable is not necessary to explain the data (a decrease in $\rho$ decreases the unconditional variance of $x$ ). Because the distribution of $\rho$ is shifted toward lower values, the distribution of $\beta$ is shifted toward higher values (see Section 3.2 and Table 1) leading to higher Bayes factors. However, while the exact likelihood does lead to higher Bayes factors, both sets of likelihood functions imply similar time series patterns. Thus the use of the exact likelihood function, by itself, is not the main driver of 
the difference between the non-stochastic and benchmark case.

We next consider the effect of a prior on the $R^{2}$ versus a prior on $\beta$. We wish to isolate the effect of the prior on $\beta$ as much as possible, so we do not want to simply compare our benchmark prior with the non-stochastic prior as these differ not only in the prior for $\beta$ but along a number of other dimensions as well.

We consider the following assumptions on the prior for $\beta$ :

$$
p\left(\beta \mid b_{0}, \Sigma, H_{1}\right) \sim N\left(0, \hat{\sigma}_{\beta}^{2}\right)
$$

where

$$
\hat{\sigma}_{\beta}=\sigma_{\eta} \hat{\sigma}_{x}^{-1} \hat{\sigma}_{u}
$$

We compute $\hat{\sigma}_{u}$ as the standard deviation of the residual from OLS regression of the predictive regression. We assume a standard uninformative prior for the remaining parameters (Zellner (1996)):

$$
p\left(b_{0}, \Sigma \mid H_{1}\right)=p\left(b_{0}, \Sigma \mid H_{0}\right) \propto|\Sigma|^{-\frac{3}{2}},
$$

for $\rho \in(-1,1)$, and zero otherwise. It follows that

$$
p\left(b_{1}, \Sigma \mid H_{1}\right) \propto \frac{1}{\sqrt{2 \pi \hat{\sigma}_{\beta}^{2}}}|\Sigma|^{-\frac{3}{2}} \exp \left\{-\frac{1}{2} \beta^{2} \hat{\sigma}_{\beta}^{-2}\right\} .
$$

In what follows, we refer to these as empirical Bayes priors. These contrast with the full Bayes priors that form our benchmark specification.

The empirical Bayes prior has several advantages for the purpose of our comparison. First, the prior over $\beta$ implied by (27) and (28) is almost identical to the conjugate- $g$ prior over $\beta .{ }^{16}$ Second, when we do not restrict $\rho$ to be between -1 and 1 and use the conditional likelihood, the results are nearly identical to the non-stochastic case (results available from the authors). This is not surprising: the prior over $\beta$ is nearly the same in both cases, and the likelihood function is exactly the same.

\footnotetext{
${ }^{16}$ The only difference is whether $\sigma_{u}$ is taken from the sample or conditioned on. Because $\sigma_{u}$ is estimated very precisely, this distinction makes little practical difference.
} 
Thus the empirical Bayes priors are similar to the priors in the non-stochastic case in many respects. However, we can impose stationarity on these priors, which we cannot do in the non-stochastic case. Thus we can make the empirical Bayes case more comparable to our benchmark case. Panel B shows the results of this exercise: We use the conditional likelihood, and compare the results of the full Bayes (benchmark) and the empirical Bayes priors. For the empirical Bayes priors, we assume $\rho \in(-1,1)$. The results in Panel B show that, while the use of empirical Bayes raises the Bayes factors somewhat, the effect is relatively small. Replacing full Bayes with empirical Bayes partially cancels out the effect of replacing the exact likelihood with the conditional likelihood, in this sample at least. Thus the use of a prior over the $R^{2}$ rather than a prior over $\beta$ plays at most a minor role in our results.

Finally, in the last panel, we consider the empirical Bayes prior and conditional likelihood with and without stationary. Without stationarity, we are in effect back to the non-stochastic regressor case. We see that whether $\rho$ is restricted to be less than one makes a large difference in the results. As explained in the previous section, the model interprets the rise in the dividend-price ratio as occurring because of an unusual sequence of negative shocks. Because of the negative correlation between the dividend-price ratio and returns, one would expect positive shocks to returns to follow negative shocks to the dividend-price ratio. In such a sample, OLS would be biased downward, not upward. However, the no-predictability model by definition implies that OLS must be biased upward. Restating somewhat, over this period there is still a negative relation between the lagged dividend-price ratio and returns. The fact that this relation is weakened is not so much evidence against predictability but rather a consequence of an unusual set of shocks. If there truly were no predictability, it would have had to have weakened much further.

It might seem that the empirical Bayes approach, or indeed the stochastic regressor approach (these are nearly identical), is more robust, as it does not require an assumption of stationarity. However, recall that these approaches rely on incorrect conditioning: They assume not only that the agent can see part of the data but not the rest, but that the agent 
is not allowed to make use of this data for inference. This seems unattractive. Moreover, this apparent robustness is itself concerning. The non-stochastic regressor approach can be shown to be equivalent to the use of ordinary least squares (OLS). ${ }^{17}$ Yet, OLS is known to be biased in the time series setting, and invalid when the right-hand-side variable is non-stationary. The fact that OLS (with its known flaws) plays a central role in the non-stochastic case, combined with the fact that this case relies on incorrect conditioning would seem to make the non-stochastic case a less than ideal foundation for Bayesian inference in time-series setting.

Rather than relying on the non-stochastic case, one could generalize the prior distribution that we introduce to allow for a non-stationary distribution for $x_{t}$. This would of course admit the possibility that excess returns, too, are non-stationary and the equity premium undefined. We leave this interesting topic to future work.

\subsection{The training sample approach}

An alternative approach that (like the non-stochastic case) makes use of the principles of conjugacy is to form a prior using a training sample. ${ }^{18}$ Unlike the non-stochastic case described in Section 3.5, the training sample approach does not require foreknowledge of the time series of $x .^{19}$

In this section, we evaluate this approach in the setting of model uncertainty. Consider a training sample (an early sub-sample of the data) with $\tilde{T}$ time series observations. Let $\tilde{X}$ and $\tilde{Y}$ denote the analog to (2.3.1) over this prior sample, $\tilde{b}_{1}$ the regression coefficients

\footnotetext{
${ }^{17}$ An apparent alternative would be to allow a flat prior for both $\beta$ and $\rho$ (thus making the prior over the $R^{2}$ unnecessary). As discussed above, this leads to Bayes factors close to zero because of Bartlett's paradox. A second alternative would be to create a training sample. We explore this alternative in detail in the next section.

${ }^{18}$ See Johannes, Korteweg, and Polson (2012).

${ }^{19}$ Though it does use the conditional rather than the exact likelihood.
} 
computed over this sample, and $\tilde{S}$ the sum of squared errors. That is:

$$
\begin{aligned}
\tilde{B}_{1} & =\left(\tilde{X}^{\top} \tilde{X}\right)^{-1} \tilde{X}^{\top} \tilde{Y} \\
\tilde{b}_{1} & =\operatorname{vec}\left(\tilde{B}_{1}\right) \\
\tilde{S} & =\left(\tilde{Y}-\tilde{X} \tilde{B}_{1}\right)^{\top}\left(\tilde{Y}-\tilde{X} \tilde{B}_{1}\right) .
\end{aligned}
$$

Prior beliefs are given as follows:

$$
\begin{aligned}
p\left(b_{1} \mid \Sigma, H_{1}\right) & \propto|\Sigma|^{-1} \exp \left\{-\frac{1}{2}\left(b_{1}-\tilde{b}_{1}\right)^{\top}\left(\Sigma^{-1} \otimes X^{\top} X\right)\left(b_{1}-\tilde{b}_{1}\right)\right\} \\
p\left(\Sigma \mid H_{1}\right) & \propto|\Sigma|^{-\frac{N+1}{2}} \exp \left\{-\frac{1}{2} \operatorname{tr} \Sigma^{-1} \tilde{S}\right\}
\end{aligned}
$$

which implies

$$
\begin{aligned}
b_{1} & \sim N\left(\tilde{b}, \Sigma \otimes\left(\tilde{X}^{\top} \tilde{X}\right)^{-1}\right) \\
\Sigma & \sim I W(\tilde{S}, \tilde{T}-2)
\end{aligned}
$$

This prior distribution can be interpreted as the beliefs the investor would have if starting with a (true) uninformative prior and updated using the conditional likelihood (14) for $\tilde{T}$ observations. The resulting distributions follow from calculations in Zellner (1996, pp. 224$227)^{20}$

Bayes theorem and the results in Zellner (1996) imply that the posterior distribution takes the same form, but with the training sample quantities replaced by their full-sample counterparts. $^{21}$ Let $^{2}$

$$
\begin{aligned}
\hat{b}_{1} & =\operatorname{vec}\left(\hat{B}_{1}\right) \\
\hat{B}_{1} & =\left(X^{\top} X\right)^{-1} X^{\top} Y \\
S & =\left(Y-X \hat{B}_{1}\right)^{\top}\left(Y-X \hat{B}_{1}\right) .
\end{aligned}
$$

\footnotetext{
${ }^{20}$ We make the standard assumption that true uninformative prior is flat for $b_{1}$ and proportional to $|\Sigma|^{-3 / 2}$ for $\Sigma$. Equations (31-37) then follow from the calculations in Zellner (1996) for the posterior given data $\tilde{X}$ and $\tilde{Y}$ of sample length $\tilde{T}$.

${ }^{21}$ For consistency with earlier sections of the paper, we continue to use $T$ as the length of the full sample. The full sample is then comprised of the training sample of length $\tilde{T}$ and an additional sample of length $T-\tilde{T}$.
} 
It follows that

$$
\begin{aligned}
p\left(b_{1} \mid \Sigma, H_{1}, D\right) & \propto|\Sigma|^{-1} \exp \left\{-\frac{1}{2}\left(b_{1}-\hat{b}_{1}\right)^{\top}\left(\Sigma^{-1} \otimes X^{\top} X\right)\left(b_{1}-\hat{b}_{1}\right)\right\} \\
p\left(\Sigma \mid H_{1}, D\right) & \propto|\Sigma|^{-\frac{T+1}{2}} \exp \left\{-\frac{1}{2} \operatorname{tr} \Sigma^{-1} S\right\}
\end{aligned}
$$

which implies

$$
\begin{aligned}
& b_{1} \sim N\left(\hat{b}_{1}, \Sigma \otimes\left(X^{\top} X\right)^{-1}\right) \\
& \Sigma \sim I W(S, T-2) .
\end{aligned}
$$

Appendix F describes the computation of Bayes factors.

The disadvantage of this approach is that inference is very sensitive to the choice of the training sample. Figure 7 shows the implied prior distribution for the coefficient $\beta$ under different training samples (Panel A) and the corresponding posterior probabilities of predictability (Panel B). We consider priors of length 8, 16 and 40 quarters (Johannes, Korteweg, and Polson (2012) use monthly data and a training sample length of 24 months). All three prior-likelihood combinations use exactly the same data; the only difference is whether the data is labeled as part of the prior or the likelihood. Nonetheless, the differences in the economic conclusions are striking. A prior formed using 8 quarters of data yields a posterior probability of only $10 \%$ at the end of the sample, while assigning 16 quarters to the prior implies a posterior probability of above $50 \%$. Increasing the data in the prior is no guarantee of stability: the posterior probability formed when the prior is 40 quarters is close to $30 \%$.

What is the source of this indeterminacy? As we discuss in Section 3.2, Bartlett's paradox implies that too diffuse a prior will lead to very low Bayes factors, because the mass of the prior is far from what the data suggest. Priors based on a small training sample run into exactly this problem (as can be seen from the prior formed using 8 quarters of data). On the other hand, using a moderate-sized training sample creates its own problems. For example, 40 quarters of data implies a prior distribution that is no longer diffuse. However, because this prior is centered at a different value than data from the full sample imply, the posterior 
probability is also lower than for the 16-quarter prior. Indeed, Figure 7 shows that the shortest training sample implies a prior that is diffuse and has little weight on $\beta=0$ while the longest training sample implies a prior that is highly informative, but also places little weight on $\beta=0$. In both cases, the Bayes factors are low.

More intuition can be obtained using the formula for the log Bayes factor that applies in this instance:

$$
\log \mathcal{B}_{10}=\log p\left(\beta=0 \mid H_{1}\right)-\log p\left(\beta=0 \mid D, H_{1}\right)
$$

(see Verdinelli and Wasserman (1995)). By definition, altering the end point of the training sample has no effect on the posterior probability of $\beta=0$, because the posterior is invariant to the how the data are divided between the training and the actual sample. However, it will of course affect the prior probability that $\beta=0$. Equation (42) shows that the log Bayes factor undergoes a linear shift depending on the training sample. Thus, while the training sample approach avoids some problems with the conjugate prior, it introduces a new one, namely: indeterminacy with respect to the choice of the training sample.

\subsection{Out-of-sample performance}

Goyal and Welch (2008) argue that the out-of-sample performance of predictive regressions, when implemented using standard techniques, is quite poor. This raises the question of whether our approach to predictability leads to superior out-of-sample performance.

In this section, we answer this question using the same CRRA utility function used to evaluate in-sample performance in Section 3.4. As in that section, we consider a one-period investor who chooses a weight in the risky asset. We first assume that the investor follows an optimal strategy, that is, he computes expected utility with respect to the predictive distribution of returns (see Section 3.4), and chooses a portfolio strategy to maximize this expected utility. We then compute the out-of-sample certainty equivalent return (CER) associated with this strategy. That is, for each quarter in the sample, we apply the optimal weights computed using information available at that quarter to the actual returns realized 
over the next quarter. This gives us a time series of quarterly returns; we use this time series to compute the expectation on the right hand side of (24).

We compare the resulting CER to that resulting from a sub-optimal strategy. ${ }^{22}$ Motivated by the findings of Goyal and Welch (2008), we first consider the strategy in which the investor computes the distribution of returns assuming no predictability and that the mean and volatility are given by their sample moments.

The results are shown in Panel A of Table 3. We find a positive difference between CERs, indicating superior out-of-sample performance relative to the sample means, for each of the prior beliefs we consider. As elsewhere in the paper, we consider a range of prior beliefs on predictability $q$ and the probability that the $R^{2}$ exceeds 0.01 . The results are largest for those prior beliefs that lead to a relatively high weight on the predictability model (namely $\left.P\left(R^{2}>.01 \mid H_{1}\right)=0.50\right)$.

Panel A of Table 3 show that strategies implied by our method outperform a simple strategy based on sample moments. We now assess the statistical significance of this outperformance. That is, we ask: could this outperformance have occurred in a sample with no predictability? Note that outperformance in a no-predictability setting need not be spurious. This is because our strategies not only incorporate evidence on predictability, but allow for Bayesian updating on all of the parameters. In performing this exercise, we are assessing the extent to which this outperformance itself constitutes evidence for return predictability. ${ }^{23}$

To accurately capture non-standard features of the portfolio return series, we simulate 400 samples under the null hypothesis of no predictability. ${ }^{24}$ For each of these samples, we calculate out-of-sample performance, repeating the procedure we used to calculate perfor-

\footnotetext{
${ }^{22}$ As in Section 3.4 and in Kandel and Stambaugh (1996), we measure utility loss by taking the difference between the CER of the optimal strategy and the CER of the suboptimal strategy.

${ }^{23}$ Unlike the rest of the paper, this exercise is purely frequentist in nature. The Bayesian investor would not require such evidence under our framework.

${ }^{24}$ In setting the parameters for this Monte Carlo, we take into account the bias in $\rho$. We choose $\rho$ to be 0.997 , which happens to be its estimate under the no-predictability model. This value of $\rho$ leads to an average OLS estimate of 0.973 , similar to that in the data.
} 
mance in actual data. We limit the number of samples to 400 due to the heavy computational requirement of this exercise. Because we have no reason to believe that our method would perform worse under the alternative hypothesis of predictability than under the null, we consider a one-tailed test and report, in brackets, the 95 percent critical value from our simulations. The results show that our out-of-sample values exceed this critical value for 11 out of the 20 priors that we consider. We conclude therefore that the out-of-sample performance our strategies exhibit would have been quite unlikely in an setting with no predictability.

Our results based on sample means raise the question of whether our strategies outperform those constructed using OLS estimates (which were used for evaluation by Goyal and Welch (2008)). We repeat the exercise above, but rather than consider a sub-optimal strategy based on sample means, we consider a sub-optimal strategies constructed using the OLS estimates. Panel B indicates that the OLS strategies do perform worse, reconciling our findings with those of Goyal and Welch. For completeness, we also report the 95 percent critical value, constructed as described above. However, there is no reason to expect that the difference between our strategies and those based on OLS would be statistically significant, and indeed they are not. ${ }^{25}$

Figure 8 shows the portfolio weights corresponding to the optimal strategy, the sample mean strategy and the OLS-based strategy. Not surprisingly, the sample mean strategy varies slowly over the period, reflecting changes in the measurement of the mean return. This strategy makes no use of the predictability of stock returns, which, when applied in our Bayesian setting, do turn out to lead to superior out of sample performance. However, the weights implied by the strategy with a $50 \%$ prior belief in predictability are notably less volatile than an OLS-based strategy. In fact, the OLS strategy spends much of the time at either $0 \%$ or $100 \%$ in equities (the discrete-time CRRA investor would never choose to

\footnotetext{
${ }^{25}$ In a previous study (Wachter and Warusawitharana (2009)) we found extremely poor performance for an OLS investor. In that study, we assumed mean-variance weights, which allowed for positions of unlimited size. In this study, we assume a CRRA investor, whose weight in the risky asset always falls between 0 and 1. This makes a difference for the OLS strategy, given the extreme nature of the implied beliefs.
} 
short equities or to invest more than $100 \%$ in equities because of the non-zero probability of negative wealth). It is likely that the Bayesian strategy would outperform by an even greater extent if one were to restrict the return distribution to allow for optimal strategies outside of these bounds. These results show how economically motivated prior beliefs can improve investment performance out-of-sample, as well as in-sample.

\subsection{Allowing for time-varying volatilities}

Stochastic volatility is a well-established property of financial returns. Here, we discuss how our approach would generalize to allow for this property.

A critical aspect to our approach is the presence of an informative prior over the predictive coefficient $\beta$. This informative prior is what allows us to calculate Bayes factors, and posterior probabilities over models. If this prior were flat, Bartlett's paradox would lead to Bayes factors close to zero. The flat prior is in a sense informative because the predictive coefficient

can become very large, leading to implied priors on the $R^{2}$ that are unreasonable on economic grounds. The Bayes factors in this case are low, not because predictability is absent, but because the supposedly uninformative prior places too much weight on unreasonable areas of the parameter space. Our approach allows the investor to place an informative prior on the predictive coefficient in a natural and intuitive way.

This insight can be readily generalized to a setting that allows for time-varying volatility. Here, we outline one such approach. Consider a data generating process as in Section 2.1, except allow the volatility of the shocks, and potentially the predictive coefficient, to change over time. That is, we compare the predictive model

$$
r_{t+1}=\alpha+\beta_{t} x_{t}+u_{t+1}
$$

to one without predictability (2), where $x_{t}$ is given by (3), and the shocks $u_{t+1}$ and $v_{t+1}$ are governed by

$$
\left[\begin{array}{c}
u_{t+1} \\
v_{t+1}
\end{array}\right] \mid r_{t}, \ldots, r_{1}, x_{t}, \ldots, x_{0} \sim N\left(0, \Sigma_{t}\right)
$$


with

$$
\Sigma_{t}=\left[\begin{array}{cc}
\sigma_{u, t}^{2} & \sigma_{u v, t} \\
\sigma_{u v, t} & \sigma_{v, t}^{2}
\end{array}\right] .
$$

We assume that $\Sigma_{t}$ follows a multivariate stochastic process such that it is positive definite with probability one. ${ }^{26}$ Rather than prior beliefs over $\Sigma$ itself, the investor would have a prior over the hyperparameters of this process. Because second moments (as opposed to first moments) can generally be accurately measured, the precise form of these priors might not turn out to be important for the conclusions. ${ }^{27}$

As discussed above, the aspect of our approach that one would wish to preserve in this setting is the informative prior on $\beta$ and its link to the $R^{2}$ statistic. The simplest generalization would keep $\eta$ as a constant parameter with the distribution

$$
\eta \mid H_{1} \sim N\left(0, \sigma_{\eta}^{2}\right)
$$

The relation

$$
\beta_{t}=\sigma_{x, t}^{-1} \sigma_{u, t} \eta
$$

then gives the prior distribution over $\beta_{t}$. This definition assumes that time-varying parameters are part of the agent's information set at time $t$, for the purpose of the $R^{2}$ calculation. ${ }^{28}$ Regardless of time-variation in $\sigma_{u, t}$ and $\sigma_{v, t}$, this would insure that the amount of predictability remains economically reasonable. Note that the posterior means for $\beta_{t}$ could, and most likely would, vary over time.

This system could be generalized still further by allowing $\eta$ itself to vary over time, replacing (43) with priors on the hyperparameters on the process for $\eta$. This prior would allow investor to have the view that predictability could vary over time in a way that is unrelated to the variance of the predictor variable or of returns.

\footnotetext{
${ }^{26}$ The difficulties in modeling $\Sigma_{t}$ are not unique to our setting, but arise in any multivariate setting with stochastic volatility.

${ }^{27}$ See Johannes, Korteweg, and Polson (2012) for a recent Bayesian analysis of stochastic volatility in a return predictability setting.

${ }^{28}$ In this, it is analogous to our current calculation for the $R^{2}$, which conditions on the true parameters. Note that this only matters for the interpretation of the priors, not for the calculation of the priors themselves.
} 
The advantage of either of these approaches is that they would allow the investor to consider both time-varying first and second moments in her investment decision. Given the evidence that second moments vary, this would be useful in improving out of sample performance. However, the qualitative findings of the importance of predictability reported earlier in the manuscript do not rely on homoskedasticity but rather on the negative correlation between shocks to the dividend-price ratio and returns. Thus, while introducing time-varying second moments would be interesting, we expect that our main results would be unaffected.

\section{Conclusion}

This study takes a Bayesian approach to the question of whether the equity premium varies over time. We consider investors who face uncertainty both over whether predictability exists, and over the strength of predictability if it does exist. We find substantial evidence in favor of predictability when the dividend-price ratio is used to predict returns. Moreover, we find large certainty equivalent losses from failing to time the market, even for investors who have strong prior beliefs in a constant equity premium. Our strategies exhibit improved outof-sample performance when compared with no-predictability strategies and when compared with OLS.

We depart from previous studies in that we model the regressor as stochastic rather than fixed. We show that this raises the probability of predictability in general, and particularly during the 2000-2005 period. Thus the way in which the regressor is modeled can significantly affect Bayesian inference, often in non-obvious ways. In this study, we model the predictive variable as following a stationary process, and the predictor variable and returns as homoskedastic. Exploring alternative distributional assumptions and their consequences for inference on returns is an interesting topic for further work. 


\section{Appendix}

\section{A Jeffreys prior under $H_{0}$}

Our derivation for the limiting Jeffreys prior on $b_{0}, \Sigma$ generalizes that of Stambaugh (1999). Zellner (1996, pp. 216-220) derives a limiting Jeffreys prior by applying (A.1) to the likelihood (17) and retaining terms of the highest order in T. Stambaugh shows that Zellner's approach is equivalent to applying (A.1) to the conditional likelihood (16), and taking the expectation in (A.1) assuming that $x_{0}$ is multivariate normal with mean (6) and variance (7). We adopt this approach.

Given a set of parameters $\mu$, data $D$, and a log-likelihood $l(\mu ; D)$, the limiting Jeffreys prior satisfies

$$
p(\mu) \propto\left|-E\left(\frac{\partial^{2} l}{\partial \mu \partial \mu^{\top}}\right)\right|^{1 / 2} .
$$

We derive the prior density for $p\left(b_{0}, \Sigma^{-1}\right)$ and then transform this into the density for $p\left(b_{0}, \Sigma\right)$ using the Jacobian. Let

$$
l_{0}\left(b_{0}, \Sigma ; D\right)=\log p\left(D \mid b_{0}, \Sigma, H_{0}, x_{0}\right)
$$

denote the natural $\log$ of the conditional likelihood. Let $\zeta=\left[\begin{array}{ll}\sigma^{(11)} & \sigma^{(12)} \sigma^{(22)}\end{array}\right]^{\top}$, where $\sigma^{(i j)}$ denotes element $(i, j)$ of $\Sigma^{-1}$. Applying (A.1) implies

$$
p\left(b_{0}, \Sigma^{-1} \mid H_{0}\right) \propto\left|-E\left[\begin{array}{cc}
\frac{\partial^{2} l_{0}}{\partial b_{0} \partial b_{0}^{\top}} & \frac{\partial^{2} l_{0}}{\partial b_{0} \partial \zeta^{\top}} \\
\frac{\partial^{2} l_{0}}{\partial \zeta \partial b_{0}^{\top}} & \frac{\partial^{2} l_{0}}{\partial \zeta \partial \zeta^{\top}}
\end{array}\right]\right|^{1 / 2} .
$$

The form of the conditional likelihood implies that

$$
l_{0}\left(b_{0}, \Sigma ; D\right)=-\frac{T}{2} \log |2 \pi \Sigma|-\frac{1}{2}\left(z-Z_{0} b_{0}\right)^{\top}\left(\Sigma^{-1} \otimes I_{T}\right)\left(z-Z_{0} b_{0}\right) .
$$

It follows from (A.4) that

$$
\frac{\partial l_{0}}{\partial b_{0}}=\frac{1}{2} Z_{0}^{\top}\left(\Sigma^{-1} \otimes I_{T}\right)\left(z-Z_{0} b_{0}\right)
$$


and

$$
\begin{aligned}
\frac{\partial^{2} l_{0}}{\partial b_{0} \partial b_{0}^{\top}} & =-\frac{1}{2} Z_{0}^{\top}\left(\Sigma^{-1} \otimes I_{T}\right) Z_{0} \\
& =-\frac{1}{2}\left[\begin{array}{cc}
\iota_{T}^{\top} & 0 \\
0 & X^{\top}
\end{array}\right]\left(\Sigma^{-1} \otimes I_{T}\right)\left[\begin{array}{cc}
\iota_{T} & 0 \\
0 & X
\end{array}\right] \\
& =-\frac{1}{2}\left[\begin{array}{cc}
\sigma^{(11)} T & \sigma^{(12)} \iota^{\top} X \\
\sigma^{(12)} X^{\top} \iota & \sigma^{(22)} X^{\top} X
\end{array}\right] .
\end{aligned}
$$

Taking the expectation conditional on $b_{0}$ and $\Sigma$ implies

$$
E\left[\frac{\partial^{2} l_{0}}{\partial b_{0} \partial b_{0}^{\top}}\right]=-\frac{T}{2}\left[\sigma^{(12)}\left[\begin{array}{c}
1 \\
\mu_{x}
\end{array}\right] \quad \sigma^{(22)}\left[\begin{array}{cc}
\sigma^{(12)}[1 & \left.\mu_{x}\right] \\
\mu_{x} & \sigma_{x}^{2}+\mu_{x}^{2}
\end{array}\right]\right]
$$

Using arguments in Stambaugh (1999), it can be shown that

$$
E\left[\frac{\partial^{2} l_{0}}{\partial b_{0} \partial \zeta^{\top}}\right]=0
$$

Moreover,

$$
-\left|E\left(\frac{\partial^{2} l_{0}}{\partial \zeta \partial \zeta^{\top}}\right)\right|=\left|\frac{\partial^{2} \log |\Sigma|}{\partial \zeta \partial \zeta^{\top}}\right|=|\Sigma|^{3}
$$

(see Box and Tiao (1973, pp. 474-475)). Therefore

$$
p\left(b_{0}, \Sigma^{-1} \mid H_{0}\right) \propto|\Phi|^{\frac{1}{2}}|\Sigma|^{\frac{3}{2}}
$$

where

$$
\Phi=\left[\begin{array}{cc}
\Sigma^{-1} & \mu_{x}\left[\begin{array}{c}
\sigma^{(12)} \\
\sigma^{(22)}
\end{array}\right] \\
\mu_{x}\left[\sigma^{(12)} \sigma^{(22)}\right] & \left(\sigma_{x}^{2}+\mu_{x}^{2}\right) \sigma^{(22)}
\end{array}\right] .
$$

This matrix $\Phi$ has the same determinant as $-E\left[\frac{\partial^{2} l_{0}}{\partial b_{0} \partial b_{0}^{\top}}\right]$ because 2 columns and 2 rows have been reversed.

From the formula for the determinant of a partitioned matrix, it follows that

$$
|\Phi|=\left|\Sigma^{-1}\right|\left|\left(\sigma_{x}^{2}+\mu_{x}^{2}\right) \sigma^{(22)}-\mu_{x}^{2}\left[\sigma^{(12)} \sigma^{(22)}\right] \Sigma\left[\begin{array}{c}
\sigma^{(12)} \\
\sigma^{(22)}
\end{array}\right]\right| .
$$


Because

$$
\Sigma\left[\begin{array}{l}
\sigma^{(12)} \\
\sigma^{(22)}
\end{array}\right]=\left[\begin{array}{l}
0 \\
1
\end{array}\right],
$$

it follows that

$$
\begin{aligned}
|\Phi| & =\left|\Sigma^{-1}\right|\left|\left(\sigma_{x}^{2}+\mu_{x}^{2}\right) \sigma^{(22)}-\mu_{x}^{2} \sigma^{(22)}\right| \\
& =|\Sigma|^{-1} \sigma_{x}^{2} \sigma^{(22) .} .
\end{aligned}
$$

The determinant of $\Sigma$ equals

$$
|\Sigma|=\sigma_{u}^{2}\left(\sigma_{v}^{2}-\sigma_{u v}^{2} \sigma_{u}^{-2}\right),
$$

while $\sigma^{(22)}=\left(\sigma_{v}^{2}-\sigma_{u v}^{2} \sigma_{u}^{-2}\right)^{-1}$. Therefore,

$$
|\Phi|=|\Sigma|^{-2} \sigma_{u}^{2} \sigma_{x}^{2}
$$

Substituting into (A.7),

$$
p\left(b_{0}, \Sigma^{-1} \mid H_{0}\right) \propto|\Sigma|^{\frac{1}{2}} \sigma_{u} \sigma_{x} .
$$

The Jacobian of the transformation from $\Sigma^{-1}$ to $\Sigma$ is $|\Sigma|^{-3}$. Therefore,

$$
p\left(b_{0}, \Sigma \mid H_{0}\right)=|\Sigma|^{-\frac{5}{2}} \sigma_{u} \sigma_{x} .
$$

\section{B Sampling from Posterior Distributions}

This section describes how to sample from the posterior distributions for our benchmark and related models. In all cases, the sampling procedure for the posteriors under $H_{1}$ and $H_{0}$ involve the Metropolis-Hastings algorithm. Below we describe the case of the exact likelihood and full Bayes prior in detail. The procedures for the conditional likelihood and for the empirical Bayes prior are similar.

\section{B.1 Posterior distribution under $H_{0}$}

Substituting (8) and (17) into (18) implies that

$$
p\left(b_{0}, \Sigma \mid H_{0}, D\right) \propto \sigma_{u}|\Sigma|^{-\frac{T+5}{2}} \exp \left\{-\frac{1}{2} \sigma_{x}^{-2}\left(x_{0}-\mu_{x}\right)^{2}-\frac{1}{2}\left(z-Z_{0} b_{0}\right)^{\top}\left(\Sigma^{-1} \otimes I_{T}\right)\left(z-Z_{0} b_{0}\right)\right\} .
$$


This posterior does not take the form of a standard density function because of the term in the likelihood involving $x_{0}$ (note that $\sigma_{x}^{2}$ is a nonlinear function of $\rho$ and $\sigma_{v}$ ). However, we can sample from the posterior using the Metropolis-Hastings algorithm.

The Metropolis-Hastings algorithm is implemented "block-at-a-time", by repeatedly sampling from $p\left(\Sigma \mid b_{0}, H_{0}, D\right)$ and from $p\left(b_{0} \mid \Sigma, H_{0} D\right)$. To calculate a proposal density for $\Sigma$, note that

$$
\left(z-Z_{0} b_{0}\right)^{\top}\left(\Sigma^{-1} \otimes I_{T}\right)\left(z-Z_{0} b_{0}\right)=\operatorname{tr}\left[\left(Y-X B_{0}\right)^{\top}\left(Y-X B_{0}\right)^{\Sigma^{-1}}\right]
$$

where

$$
B_{0}=\left[\begin{array}{ll}
\alpha & \theta \\
0 & \rho
\end{array}\right]
$$

The proposal density for the conditional probability of $\Sigma$ is the inverted Wishart with $T+2$ degrees of freedom and scale factor of $\left(Y-X B_{0}\right)^{\top}\left(Y-X B_{0}\right)$. The target is therefore

$$
p\left(\Sigma \mid b_{0}, H_{0}, D\right) \propto \sigma_{u} \exp \left\{-\frac{1}{2}\left(x_{0}-\mu_{x}\right)^{2} \sigma_{x}^{-2}\right\} \times \text { proposal. }
$$

Let

$$
V_{0}=\left(Z_{0}^{\top}\left(\Sigma^{-1} \otimes I_{T}\right) Z_{0}\right)^{-1}
$$

Let

$$
\hat{b}_{0}=V_{0} Z_{0}^{\top}\left(\Sigma^{-1} \otimes I_{T}\right) z
$$

It follows from completing the square that

$$
\left(z-Z_{0} b_{0}\right)^{\top}\left(\Sigma^{-1} \otimes I_{T}\right)\left(z-Z_{0} b_{0}\right)=\left(b_{0}-\hat{b}_{0}\right)^{\top} V_{0}^{-1}\left(b_{0}-\hat{b}_{0}\right)+\text { terms independent of } b_{0} .
$$

The proposal density for $b_{0}$ is therefore multivariate normal with mean $\hat{b}_{0}$ and variancecovariance matrix $V_{0}$. The accept-reject algorithm of Chib and Greenberg (1995, Section 5) is used to sample from the target density, which is equal to

$$
p\left(b_{0} \mid \Sigma, H_{0}, D\right) \propto \exp \left\{-\frac{1}{2}\left(x_{0}-\mu_{x}\right)^{2} \sigma_{x}^{-2}\right\} \times \text { proposal. }
$$

Note that $\sigma_{u}$ and $\Sigma$ are in the constant of proportionality. Drawing successively from the conditional posteriors for $\Sigma$ and $b_{0}$ produces a density that converges to the full posterior conditional on $H_{0}$. 


\section{B.2 Posterior distribution under $H_{1}$}

Substituting (12) and (15) into (18) implies that

$$
\begin{aligned}
p\left(b_{1}, \Sigma \mid H_{1}, D\right) \propto \sigma_{x}|\Sigma|^{-\frac{T+5}{2}} \exp \left\{-\frac{1}{2} \beta^{2}\left(\sigma_{\eta}^{2} \sigma_{x}^{-2} \sigma_{u}^{2}\right)^{-2}-\frac{1}{2} \sigma_{x}^{-2}\left(x_{0}-\mu_{x}\right)^{2}\right\} \\
\exp \left\{-\frac{1}{2}\left(z-Z_{1} b_{1}\right)^{\top}\left(\Sigma^{-1} \otimes I_{T}\right)\left(z-Z_{1} b_{1}\right)\right\} .
\end{aligned}
$$

The sampling procedure is similar to that described in Appendix B.1. Details can be found in Wachter and Warusawitharana (2009). To summarize, we first draw from the posterior $p\left(\Sigma \mid b_{1}, H_{1}, D\right)$. The proposal density is an inverted Wishart with $T+2$ degrees of freedom and scale factor $\left(Y-X B_{1}\right)^{\top}\left(Y-X B_{1}\right)$, where

$$
B_{1}=\left[\begin{array}{ll}
\alpha & \theta \\
\beta & \rho
\end{array}\right]
$$

We then draw from $p\left(\theta, \rho \mid \alpha, \beta, \Sigma, H_{1}, D\right)$. The proposal density is multivariate normal with mean and variance determined by the conditional normal distribution. Finally, we draw from $p\left(\alpha, \beta \mid \theta, \rho, \Sigma, H_{1}, D\right)$. In this case, the target and the proposal are the same, and are also multivariate normal.

\section{Computing the Bayes factor}

This section describes computation of Bayes factors for the benchmark and related models. Verdinelli and Wasserman (1995) show

$$
\mathcal{B}_{10}^{-1}=p\left(\beta=0 \mid H_{1}, D\right) E\left[\frac{p\left(b_{0}, \Sigma \mid H_{0}\right)}{p\left(\beta=0, b_{0}, \Sigma \mid H_{1}\right)} \mid \beta=0, H_{1}, D\right] .
$$

To compute $p\left(\beta=0 \mid H_{1}, D\right)$, note that

$$
p\left(\beta=0 \mid H_{1}, D\right)=\int p\left(\beta=0 \mid b_{0}, \Sigma, H_{1}, D\right) p\left(b_{0}, \Sigma \mid H_{1}, D\right) d b_{0} d \Sigma
$$

As discussed in Appendix B.2, the posterior distribution of $\alpha$ and $\beta$ conditional on the remaining parameters is normal. We can therefore compute $p\left(\beta=0 \mid b_{0}, \Sigma, H_{1}, D\right)$ in 
closed form by using the properties of the conditional normal distribution. Consider $N$ draws from the full posterior: $\left(\left(b_{1}^{(1)}, \Sigma^{(1)}\right), \ldots,\left(b_{1}^{(N)}, \Sigma^{(N)}\right)\right)$, where we can write $\left(b_{1}^{(i)}, \Sigma^{(i)}\right)$ as $\left(\beta^{(i)}, b_{0}^{(i)}, \Sigma^{(i)}\right)$. We use these draws to integrate out over $b_{0}$ and $\Sigma$. It follows from (C.2) that

$$
p\left(\beta=0 \mid H_{1}, D\right) \approx \frac{1}{N} \sum_{i=1}^{N} p\left(\beta=0 \mid b_{0}^{(i)}, \Sigma^{(i)}, H_{1}, D\right),
$$

where the approximation is accurate for large $N$.

To compute the second term in (C.1), we observe that

$$
\frac{p\left(b_{0}, \Sigma \mid H_{0}\right)}{p\left(\beta=0, b_{0}, \Sigma \mid H_{1}\right)}=\frac{p\left(b_{0}, \Sigma \mid H_{0}\right)}{p\left(\beta=0 \mid b_{0}, \Sigma, H_{1}\right) p\left(b_{0}, \Sigma \mid H_{1}\right)}=\sqrt{2 \pi} \sigma_{\beta},
$$

because $p\left(b_{0}, \Sigma \mid H_{0}\right)=p\left(b_{0}, \Sigma \mid H_{1}\right)$. Note that $\sigma_{\beta}=\sigma_{\eta} \sigma_{x}^{-1} \sigma_{u}$. We require the expectation taken with respect to the posterior distribution conditional on the existence of predictability and the realization $\beta=0$. To calculate this expectation, we $\operatorname{draw}\left(\left(b_{0}^{(1)}, \Sigma^{(1)}\right), \ldots,\left(b_{0}^{(N)}, \Sigma^{(N)}\right)\right)$ from $p\left(b_{0}, \Sigma \mid \beta=0, H_{1}, D\right)$. This involves modifying the procedure for drawing from the posterior for $b_{1}, \Sigma$ given $H_{1}$ (see Appendix B.2). We sample from $p\left(\Sigma \mid \alpha, \beta=0, \theta, \rho, H_{1}, D\right)$, then from $p\left(\rho, \theta \mid \alpha, \beta=0, \Sigma, H_{1}, D\right)$ and finally from $p\left(\alpha \mid \beta=0, \Sigma, \theta, \rho, H_{1}, D\right)$, and repeat until the desired number of draws are obtained. All steps except the last are identical to those described in Appendix B.2 (the value of $\beta$ is identically zero rather than the value from the previous draw). For the last step we derive $p\left(\alpha \mid \beta=0, \Sigma, \theta, \rho, H_{1}, D\right)$ from the joint distribution $p\left(\alpha, \beta \mid \Sigma, \theta, \rho, H_{1}, D\right)$, making use of the properties of the conditional normal distribution.

Given these draws from the posterior distribution, the second term equals

$$
E\left[\frac{p\left(b_{0}, \Sigma \mid H_{0}\right)}{p\left(\beta=0, b_{0}, \Sigma \mid H_{1}\right)} \mid \beta=0, H_{1}, D\right] \approx \frac{1}{N} \sum_{i=1}^{N} \sqrt{2 \pi} \sigma_{\eta}\left(\sigma_{x}^{(i)}\right)^{-1} \sigma_{u}^{(i)} .
$$

\section{The posterior distribution and Bayes factor for the conjugate $g$-prior and conditional likelihood}

This section generalizes results in Zellner (1996) to the case of a multivariate regression system with an informative conjugate prior. We assume a multivariate version of the conjugate 
$g$-prior as follows:

$$
\begin{aligned}
b_{1} \mid \Sigma, H_{1} & \sim N\left(0, g^{-1}\left(\Sigma \otimes\left(X^{\top} X\right)^{-1}\right)\right), \\
\Sigma \mid H_{1} & \sim I W\left(S_{0}, N_{0}-2\right),
\end{aligned}
$$

where $g^{-1}$ is a scale parameter that determines the degree of precision of the prior, $I W$ denotes the inverse-Wishart distribution, and $N_{0}$ and $S_{0}$ can be interpreted as the length of a hypothetical no-predictability prior sample and the sum of squared errors of this sample, respectively. ${ }^{29}$

It is convenient to define

$$
B_{1}=\left[\begin{array}{ll}
\alpha & \theta \\
\beta & \rho
\end{array}\right]
$$

and to write the prior (D.4) as

Given $B_{1}$ defined as in (B.1), it follows from Zellner (1996, Eq. 8.14) that

$$
p\left(B_{1} \mid \Sigma, H_{1}\right) \propto|\Sigma|^{-1} \exp \left\{-\frac{1}{2} \operatorname{tr}\left(g B_{1}^{\top}\left(X^{\top} X\right) B_{1} \Sigma^{-1}\right)\right\} .
$$

Note that the variance of $b_{1}$ equals $\Sigma \otimes\left(X^{\top} X\right)^{-1}$, and that

$$
\left|\Sigma \otimes\left(X^{\top} X\right)^{-1}\right|^{-\frac{1}{2}} \propto|\Sigma|^{-1}
$$

because $X^{\top} X$ can be regarded as a constant when calculating the distribution of $B_{1}$. Further, the density for the inverse Wishart distribution (D.5) equals

$$
p\left(\Sigma \mid H_{1}\right) \propto|\Sigma|^{-\left(N_{0}+1\right) / 2} \exp \left\{-\frac{1}{2} \operatorname{tr}\left(\Sigma^{-1} S_{0}\right)\right\} .
$$

Therefore the joint prior is given by

$$
p\left(B_{1}, \Sigma \mid H_{1}\right)=|2 \pi \Sigma|^{-\frac{N_{0}+3}{2}} \exp \left\{-\frac{1}{2} \operatorname{tr}\left(g B_{1}^{\top}\left(X^{\top} X\right) B_{1} \Sigma^{-1}+S_{0} \Sigma^{-1}\right)\right\} .
$$

\footnotetext{
${ }^{29}$ This interpretation is consistent with having a standard uninformative "prior" before viewing this nopredictability "prior sample" of $p\left(b_{1}\right) \propto$ constant and $p(\Sigma) \propto|\Sigma|^{-3 / 2}$. See Zellner (1996, Chapter 8.1). A prior sample of length greater than 2 is necessary for a well-defined posterior distribution, since the data also need to be sufficient to identify $b_{1}$.
} 
Note that this prior imposes a particular structure on the covariance matrix of the parameters that mimics the likelihood specification. It is this structure that is responsible for this specification's tractability. Note also that the data enter into the prior through the term $\left(X^{\top} X\right)^{-1}$, so that this prior requires incorrect conditioning. The entire time path of the state variable must be known when prior beliefs are formulated.

We combine this prior with the conditional likelihood function. ${ }^{30}$ Let

$$
\begin{aligned}
\hat{B}_{1} & =\left(X^{\top} X\right)^{-1} X^{\top} Y \\
S & =\left(Y-X \hat{B}_{1}\right)^{\top}(Y-X \hat{B})
\end{aligned}
$$

Given this notation, we can rewrite (14) as follows:

$$
p\left(D \mid B_{1}, \Sigma, H_{1}\right) \propto|\Sigma|^{-\frac{T}{2}} \exp \left\{-\frac{1}{2} \operatorname{tr}\left(\left(B_{1}-\hat{B}_{1}\right)^{\top} X^{\top} X\left(B_{1}-\hat{B}_{1}\right) \Sigma^{-1}+S \Sigma^{-1}\right)\right\}
$$

where $\propto$ in (D.11) should be taken to mean that we have eliminated multiplicative terms that do not depend on $B_{1}$ and $\Sigma$. For more detail, see Zellner (1996, Chapter 8.1).

Define sufficient statistics for the posterior as follows

$$
\begin{aligned}
\bar{B}_{1} & =\left(X^{\top} X(1+g)\right)^{-1}\left(X^{\top} Y\right) \\
\bar{S} & =S_{0}+Y^{\top} Y-\left(Y^{\top} X\right)\left(X^{\top} X(1+g)\right)^{-1}\left(X^{\top} Y\right)
\end{aligned}
$$

Note that $\bar{S}$ can be rewritten as

$$
\bar{S}=S_{0}+S+\hat{B}_{1}^{\top} X^{\top} X \hat{B}_{1}-\bar{B}_{1}^{\top}\left(X^{\top} X\right)(1+g) \bar{B}_{1}
$$

Bayes rule implies that the posterior is given by

$$
p\left(B_{1}, \Sigma \mid D, H_{1}\right) \propto p\left(D \mid B_{1}, \Sigma, H_{1}\right) p\left(B_{1}, \Sigma \mid H_{1}\right)
$$

where the first and second terms on the right hand side are given by (D.11) and (D.8) respectively. Completing the square and using (D.12) implies that the posterior density

\footnotetext{
${ }^{30}$ We cannot use the exact likelihood function because the prior does not lead to a well-defined distribution for the predictor variable.
} 
equals

$$
\begin{aligned}
& p\left(B_{1}, \Sigma \mid D, H_{1}\right)= \\
& |\Sigma|^{-\frac{T+N_{0}+3}{2}} \exp \left\{-\frac{1}{2} \operatorname{tr}\left(\left(B_{1}-\bar{B}_{1}\right)^{\top}\left(X^{\top} X(1+g)\right)\left(B_{1}-\bar{B}_{1}\right) \Sigma^{-1}+\bar{S} \Sigma^{-1}\right)\right\} .
\end{aligned}
$$

We now factor the joint posterior (D.13) into a posterior for $B_{1}$ conditional on $\Sigma$ and the marginal posterior for $\Sigma$. This is an important step in computing the Bayes factor, as will be apparent in what follows. By definition,

$$
p\left(B_{1}, \Sigma \mid D, H_{1}\right)=p\left(B_{1} \mid \Sigma, D, H_{1}\right) p\left(\Sigma \mid D, H_{1}\right) .
$$

Define

$$
\bar{b}_{1}=\operatorname{vec}\left(\bar{B}_{1}\right)
$$

The factorization in (D.14) is accomplished as follows:

$$
\begin{aligned}
p\left(B_{1} \mid \Sigma, D, H_{1}\right) & \propto|\Sigma|^{-1} \exp \left\{-\frac{1}{2} \operatorname{tr}\left(\left(B_{1}-\bar{B}_{1}\right)^{\top}\left(X^{\top} X(1+g)\right)\left(B_{1}-\bar{B}_{1}\right) \Sigma^{-1}\right)\right\} \\
& =|\Sigma|^{-1} \exp \left\{-\frac{1}{2}\left(b_{1}-\bar{b}_{1}\right)^{\top}\left(\Sigma^{-1} \otimes X^{\top} X(1+g)\right)\left(b_{1}-\bar{b}_{1}\right)\right\}
\end{aligned}
$$

and

$$
p\left(\Sigma \mid D, H_{1}\right) \propto|\Sigma|^{-\frac{T+N_{0}+1}{2}} \exp \left\{-\frac{1}{2} \operatorname{tr}\left(\bar{S} \Sigma^{-1}\right)\right\}
$$

The distribution (D.15) represents a multivariate normal distribution. ${ }^{31}$

Our ultimate goal is to calculate the marginal posterior for $\beta$, which is the second element of $b_{1}$. Let $\bar{\beta}$ be the second element of $\bar{b}_{1}$ and define

$$
\bar{\nu}_{x}=\left[\left(X^{\top} X\right)^{-1}\right]_{22},
$$

namely the second diagonal element of $\left(X^{\top} X\right)^{-1} \cdot{ }^{32}$ It follows from (D.15) and properties of the multivariate normal distribution that

$$
p\left(\beta \mid \Sigma, D, H_{1}\right) \propto \frac{1}{\sigma_{u}} \exp \left\{-\frac{1}{2} \sigma_{u}^{-2}(1+g) \bar{\nu}_{x}(\beta-\bar{\beta})^{2}\right\},
$$

\footnotetext{
${ }^{31}$ As in (D.6), for (D.15) to be multivariate normal, $|\Sigma|$ must be raised to the power -1 .

${ }^{32}$ Note that this element is also equal to $T \hat{\sigma}_{x}^{2}$, namely $T$ (the number of time series observations on the return variable) multiplied by the sample variance of the predictor taken from time 0 to time $T-1$.
} 
where we have used the fact that $\left(\Sigma^{-1} \otimes\left(X^{\top} X\right)\right)^{-1}=\Sigma \otimes\left(X^{\top} X\right)^{-1}$,

Further, note that (D.17) depends only on $\sigma_{u}$. Therefore, to calculate the marginal prior for $\beta$, we only need to integrate out $\sigma_{u}$. It follows from (D.16) and properties of the inverse Wishart distribution that

$$
p\left(\sigma_{u}^{2} \mid D, H_{1}\right) \propto \frac{1}{\sigma_{u}^{T+N_{0}-1}} \exp \left\{-\frac{\bar{S}_{11}}{2 \sigma_{u}^{2}}\right\}
$$

where $\bar{S}_{11}$ is the first diagonal element of $\bar{S}$ (see Zellner (1996, p. 227-228)). It follows that

$$
\begin{aligned}
p\left(\beta \mid D, H_{1}\right) & =\int_{0}^{\infty} p\left(\beta \mid \sigma_{u}^{2}, D, H_{1}\right) p\left(\sigma_{u}^{2} \mid D, H_{1}\right) d \sigma_{u}^{2} \\
& \propto \int_{0}^{\infty} \frac{1}{\sigma_{u}^{T+N_{0}}} \exp \left\{-\frac{1}{2 \sigma_{u}^{2}}\left((1+g) \bar{\nu}_{x}(\beta-\bar{\beta})^{2}+\bar{S}_{11}\right)\right\} d \sigma_{u}^{2} \\
& \propto\left((1+g) \bar{\nu}_{x}(\beta-\bar{\beta})^{2}+\bar{S}_{11}\right)^{-\frac{T+N_{0}-2}{2}} \\
& \propto\left(1+\frac{1}{T+N_{0}-3}\left(\frac{(1+g) \bar{\nu}_{x}\left(T+N_{0}-3\right)}{\bar{S}_{11}}\right)(\beta-\bar{\beta})^{2}\right)^{-\frac{T+N_{0}-2}{2}}
\end{aligned}
$$

Therefore, $\beta$ has a $t$-distribution with location parameter $\bar{\beta}$, scale parameter

$$
\left((1+g) T \bar{\nu}_{x}\left(T+N_{0}-3\right)\right)^{-1 / 2} \bar{S}_{11}^{1 / 2}
$$

and $T+N_{0}-3$ degrees of freedom.

Under the condition

$$
p\left(b_{0}, \Sigma \mid H_{0}\right)=p\left(b_{0}, \Sigma \mid \beta=0, H_{1}\right)
$$

the Bayes factor can be computed using the marginal prior and posterior distributions for $\beta$ :

$$
\mathcal{B}_{10}=\frac{p\left(\beta=0 \mid H_{1}\right)}{p\left(\beta=0 \mid D, H_{1}\right)}
$$

(see Verdinelli and Wasserman (1995)). The value of $p\left(\beta=0 \mid D, H_{1}\right)$ can be computed based on (D.20) using the formula for the density of a $t$-distribution. We can perform the analogous calculation for the prior distribution to find

$$
p\left(\beta \mid H_{1}\right) \propto\left(1+\frac{1}{N_{0}-3}\left(\frac{g \bar{\nu}_{x}\left(N_{0}-3\right)}{S_{0,11}}\right) \beta^{2}\right)^{-\frac{N_{0}-2}{2}},
$$


where $S_{0,11}$ is the first diagonal element of $S_{0}$. This is a central $t$ distribution with scale parameter

$$
\left(g \bar{\nu}_{x}\left(N_{0}-3\right)\right)^{-1 / 2} S_{0,11}^{1 / 2}
$$

and $N_{0}-3$ degrees of freedom.

\section{E The posterior distribution and Bayes factor for the conjugate $g$-prior when the regressor is strictly ex- ogenous}

When the regressor is strictly exogenous, it is correct to use only the return equation. With some abuse of notation, let $b=[\alpha, \beta]^{\top}$. The prior distribution takes the form

$$
\begin{aligned}
p\left(b \mid \sigma_{u}, H_{1}\right) & \propto \frac{1}{\sigma_{u}^{2}} \exp \left\{-\frac{1}{2 \sigma_{u}^{2}} b^{\top}\left(g X^{\top} X\right) b\right\} \\
p\left(\sigma_{u}^{2} \mid H_{1}\right) & \propto \frac{1}{\sigma_{u}^{N_{0}}} \exp \left\{-\frac{1}{2} \sigma_{u}^{-2} s_{0}\right\}
\end{aligned}
$$

where $s_{0}$ and $N_{0}$ are constants. We can rewrite this system in terms of familiar distributions:

$$
\begin{aligned}
b \mid \sigma_{u}, H_{1} & \sim N\left(0, g^{-1} \sigma_{u}^{2}\left(X^{\top} X\right)^{-1}\right) \\
\sigma_{u}^{2} \mid H_{1} & \sim I W\left(s_{0}, N_{0}-2\right)
\end{aligned}
$$

As in the previous section, it is as if we have a "true" uninformative prior of $p\left(\sigma_{u}^{2}\right) \propto \sigma_{u}^{-2}$ and $p(b) \propto$ constant before seeing a "prior sample" with $N_{0}$ observations. Because $\sigma_{u}^{2}$ is scalar in this case, its distribution can also be characterized as an inverse-Gamma.

Define

$$
\begin{aligned}
\hat{b} & =\left(X^{\top} X\right)^{-1} X^{\top} R \\
s & =(R-X \hat{b})^{\top}(R-X \hat{b}) .
\end{aligned}
$$

Note that $s=S_{11}$ in the previous section. The likelihood function is

$$
p\left(R \mid X, b, \sigma_{u}^{2}\right) \propto \sigma_{u}^{-T} \exp \left\{-\frac{1}{2 \sigma_{u}^{2}}\left((b-\hat{b})^{\top} X^{\top} X(b-\hat{b})+s \sigma_{u}^{-2}\right)\right\}
$$


where, as in the previous section $\propto$ should be taken to mean that we have eliminated multiplicative terms that do not depend on $b$ and $\sigma_{u}$.

Analogously to the previous section, define

$$
\bar{b}=\left(X^{\top} X(1+g)\right)^{-1}\left(X^{\top} R\right)
$$

and

$$
\begin{aligned}
\bar{s} & =s_{0}+R^{\top} R-\left(Y^{\top} X\right)\left(X^{\top} X(1+g)\right)^{-1}\left(X^{\top} R\right) \\
& =s_{0}+s+\hat{b}^{\top} X^{\top} X \hat{b}-\bar{b}^{\top}\left(X^{\top} X\right)(1+g) \bar{b} .
\end{aligned}
$$

Note that if $g$ is the same, $\bar{b}$ will equal the first column of $\bar{B}_{1}$, and $\bar{s}$ will equal $\bar{S}_{11}$ (assuming that $s_{0}=S_{0,11}$. Completing the square and using (E.29) implies

$$
p\left(b, \sigma_{u}^{2} \mid R, X, H_{1}\right) \propto \sigma_{u}^{-\left(T+N_{0}+2\right)} \exp \left\{-\frac{1}{2 \sigma_{u}^{2}}\left((b-\bar{b})^{\top}\left(X^{\top} X(1+g)\right)(b-\bar{b})+\bar{s}\right)\right\} .
$$

The posterior for $b$ conditional on $\sigma_{u}$ is multivariate normal:

$$
p\left(b \mid \sigma_{u}, R, X, H_{1}\right) \propto \frac{1}{\sigma_{u}^{2}} \exp \left\{-\frac{1}{2 \sigma_{u}^{2}}(b-\bar{b})^{\top} X^{\top} X(1+g)(b-\bar{b})\right\}
$$

while the marginal distribution for $\sigma_{u}^{2}$ is inverse-Wishart (or, in this case, inverse-Gamma):

$$
p\left(\sigma_{u}^{2} \mid R, X, H_{1}\right) \propto \sigma_{u}^{-\left(T+N_{0}\right)} \exp \left\{-\frac{1}{2 \sigma_{u}^{2}} \bar{s}\right\}
$$

It follows from (E.31) and properties of the multivariate normal distribution that the distribution for $\beta$ (the second element of $b$ ) is given by

$$
p\left(\beta \mid \sigma_{u}^{2}, R, X, H_{1}\right) \propto \sigma_{u}^{-1} \exp \left\{-\frac{1}{2 \sigma_{u}^{2}}(1+g) \bar{\nu}_{x}(\beta-\bar{\beta})^{2}\right\},
$$

where $\bar{\beta}$ is the second element of $\bar{b}$. Finally, we compute

$$
\begin{aligned}
p\left(\beta \mid R, X, H_{1}\right) & \propto \int_{0}^{\infty} p\left(\beta \mid \sigma_{u}^{2}, R, X, H_{1}\right) p\left(\sigma_{u}^{2} \mid R, X, H_{1}\right) d \sigma_{u}^{2} \\
& \propto \int_{0}^{\infty} \frac{1}{\sigma_{u}^{T+N_{0}+1}} \exp \left\{-\frac{1}{2 \sigma_{u}^{2}}\left((1+g) \bar{\nu}_{x}(\beta-\bar{\beta})^{2}+\bar{s}\right)\right\} d \sigma_{u}^{2} \\
& \propto\left((1+g) \bar{\nu}_{x}(\beta-\bar{\beta})^{2}+\bar{s}\right)^{-\frac{T+N_{0}-1}{2}}
\end{aligned}
$$


Arguing by analogy with (D.20), we see that $\beta$ has a $t$-distribution with location parameter $\bar{\beta}$, scale parameter

$$
\left((1+g) \bar{\nu}_{x}\left(T+N_{0}-2\right)\right)^{-1 / 2} \bar{s}^{1 / 2}
$$

and $T+N_{0}-2$ degrees of freedom. The prior distribution for $\beta$ will be a central $t$ with scale parameter

$$
\left(g \bar{\nu}_{x}\left(N_{0}-2\right)\right)^{-1 / 2} s_{0}^{1 / 2}
$$

and $N_{0}-2$ degrees of freedom. Bayes factors can then be computed using (D.21) and (D.22).

It is instructive to compare these results with those of Appendix D. The marginal prior and posterior for $\beta$ is nearly the same in the one-equation setting as in the two-equation setting, except for the degrees of freedom in the $t$-distribution. There is an additional degree of freedom in the one-equation setting, corresponding to a $t$-distribution that is somewhat less fat-tailed. As Zellner (1996, Chapter 8.1) discusses, this change in the degrees of freedom arises because of the need to estimate an additional parameter in the two-equation case, namely the correlation between shocks to $u$ and shocks to $v$. Because, in effect, the same data needs to work harder in the two-equation case, the distributions are more diffuse. Mathematically, the difference arises from the fact that the marginal distribution of $\sigma_{u}^{2}$ in

(D.18) is not the same as the marginal distribution of $\sigma_{u}^{2}$ in the single-equation case. However, if the regressor is strictly exogenous, namely if $u$ and $v$ are assumed to be independent, the one-equation case and the two-equation case will yield identical Bayes factors, a manifestation of the general principle discussed in Section 2.5.

\section{F Bayes factors for the training sample approach}

Bayes factors for the training sample approach (described in Section 3.7) can be computed as a special case of those in Appendix D. Define

$$
\tilde{\nu}_{x}=\left[\left(\tilde{X}^{\top} \tilde{X}\right)^{-1}\right]_{22}
$$


where we use the notation of Section 3.7, namely variables with a tilde on top correspond to quantities computed over the training sample. Then the prior distribution for $\beta$ can be computed using results for the posterior distribution calculated in Appendix D, for a uninformative prior $\left(g=0, N_{0}=0\right)$, and with full-sample quantities replaced by their training sample counterparts. That is, (D.19) becomes

$$
p\left(\beta \mid H_{1}\right) \propto\left(\tilde{\nu}_{x}(\beta-\tilde{\beta})^{2}+\tilde{S}_{11}\right)^{-\frac{\tilde{T}-2}{2}}
$$

where $\tilde{\beta}$ is the second element of $\tilde{b}_{1}$ and $\tilde{S}_{11}$ is the first diagonal element of $\tilde{S}$. Similarly, the posterior can be calculated in the same way (again, $g=0$ and $N_{0}=0$ ), keeping in mind that the full-sample quantities in this case are as in OLS regression. That is (D.19) becomes

$$
p\left(\beta \mid D, H_{1}\right) \propto\left(\bar{\nu}_{x}(\beta-\hat{\beta})^{2}+S_{11}\right)^{-\frac{T-2}{2}} .
$$

The calculation of the Bayes factor of course requires the true prior and posterior densities of $\beta$ at zero, not just these values up to a constant that does not depend on $\beta$. These densities can be calculated by observing, as in Appendix D, that (F.33) and (F.34) imply $t$-distributions, with known density functions.

\section{G Results for the yield spread}

In Figure 9, we report results in which the predictor variable is the difference between the continuously-compounded 5-year zero-coupon bond yield and the yield on the 3-month Treasury Bill. Panel A shows that, while the yield spread had significant predictive power for returns in the early part of the sample, its power has been steadily declining. At the end of the sample, the posterior probability of predictability with the yield spread is about $50 \%$, close to the prior. The yield spread has a lower autocorrelation than the dividend yield, and innovations to the yield spread have low correlation with innovations to returns. Both of these facts suggest that the non-stochastic and benchmark analyses would imply very similar results, which indeed they do. 


\section{References}

Ando, Tomohiro, and Arnold Zellner, 2010, Hierarchical Bayesian analysis of the seemingly unrelated regression and simultaneous equations models using a combination of direct Monte Carlo and importance sampling techniques, Bayesian Analysis 5, 65-96.

Avramov, Doron, 2002, Stock return predictability and model uncertainty, Journal of Financial Economics 64, 423-458.

Barberis, Nicholas, 2000, Investing for the long run when returns are predictable, Journal of Finance 55, 225-264.

Bartlett, M.S., 1957, Comment on 'A Statistical Paradox' by D. V. Lindley, Biometrika 44, $533-534$.

Box, George E.P., and George C. Tiao, 1973, Bayesian Inference in Statistical Analysis. (Addison-Wesley Pub. Co. Reading, MA).

Brandt, Michael W., Amit Goyal, Pedro Santa-Clara, and Jonathan R. Stroud, 2005, A simulation approach to dynamics portfolio choice with an application to learning about return predictability, Review of Financial Studies 18, 831-873.

Campbell, John Y., 2008, Viewpoint: Estimating the equity premium, Canadian Journal of Economics 41, 1-21.

Campbell, John Y., and Robert J. Shiller, 1988, The dividend-price ratio and expectations of future dividends and discount factors, Review of Financial Studies 1, 195-228.

Campbell, John Y., and Luis M. Viceira, 1999, Consumption and portfolio decisions when expected returns are time-varying, Quarterly Journal of Economics 114, 433-495.

Campbell, John Y., and Motohiro Yogo, 2006, Efficient tests of stock return predictability, Journal of Financial Economics 81, 27-60. 
Chen, Zengjing, and Larry Epstein, 2002, Ambiguity, risk and asset returns in continuous time, Econometrica 70, 1403-1443.

Chib, Siddhartha, and Edward Greenberg, 1995, Understanding the Metropolis-Hastings algorithm, American Statistician 49, 327-335.

Chipman, Hugh, Edward I. George, and Robert E. McCulloch, 2001, The practical implementation of Bayesian model selection, in P. Lahiri, eds.: Model Selection (IMS Lecture Notes, Bethesda, MA ).

Cochrane, John H., 2008, The Dog That Did Not Bark: A Defense of Return Predictability, The Review of Financial Studies 21, 1533-1575.

Cremers, K.J. Martjin, 2002, Stock return predictability: A Bayesian model selection perspective, Review of Financial Studies 15, 1223-1249.

Dickey, James M., 1971, The weighted likelihood ratio, linear hypotheses on normal location paramaters, The Annals of Mathematical Statistics 42, 204-223.

Faust, Jon, and Jonathan H. Wright, 2011, Efficient Prediction of Excess Returns, Review of Economics and Statistics 93, 647-659.

Fernandez, Carmen, Eduardo Ley, and Mark F. J. Steel, 2001, Benchmark priors for Bayesian model averaging, Journal of Econometrics 100, 381-427.

Goyal, Amit, and Ivo Welch, 2008, A comprehensive look at the empirical performance of equity premium prediction, Review of Financial Studies 21, 1455-1508.

Hamilton, J. D., 1994, Time Series Analysis. (Oxford University Press Princeton, NJ).

Jeffreys, Harold, 1961, Theory of Probability. (Oxford University Press Clarenden).

Johannes, Michael, and Nicholas Polson, 2006, MCMC methods for financial econometrics, in Yacine Ait-Sahalia, and Lars Hansen, eds.: Handbook of Financial Econometrics (Elsevier, North-Holland ). 
Johannes, Michael, Nicholas Polson, and Jonathan R. Stroud, 2002, Sequential optimal portfolio performance: Market and volatility timing, Working paper, Columbia University, University of Chicago, and University of Pennsylvania.

Johannes, Michael S., Arthur G. Korteweg, and Nicholas G. Polson, 2012, Sequential learning, predictability, and optimal portfolio returns, fothcoming, Journal of Finance.

Kandel, Shmuel, and Robert F. Stambaugh, 1996, On the predictability of stock returns: An asset allocation perspective, Journal of Finance 51, 385-424.

Kass, R., and A. E. Raftery, 1995, Bayes factors, Journal of the American Statistical Association $90,773-795$.

Lewellen, Jonathan, 2004, Predicting returns with financial ratios, Journal of Financial Economics 74, 209-235.

Mehra, Rajnish, and Edward Prescott, 1985, The equity premium puzzle, Journal of Monetary Economics 15, 145-161.

Pastor, Lubos, and Robert F. Stambaugh, 2009, Predictive systems: Living with imperfect predictors, Journal of Finance 64, 1583 - 1628.

Poirier, Dale J., 1978, The effect of the first observation in regression models with first-order autoregressive disturbances, Journal of the Royal Statistical Society, Series C, Applied Statistics 27, 67-68.

Shanken, Jay A., and Ane Tamayo, 2011, Payout yield, risk and mispricing, a Bayesian analysis, forthcoming, Journal of Financial Economics.

Skoulakis, Georgios, 2007, Dynamic portfolio choice with Bayesian learning, Working paper, University of Maryland.

Stambaugh, Robert F., 1999, Predictive regressions, Journal of Financial Economics 54, $375-421$. 
Stock, James H., and Mark W. Watson, 2012, Generalized Shrinkage Methods for Forecasting Using Many Predictors, Journal of Business 8 Economic Statistics 30, 481-493.

Van Binsbergen, Jules H., and Ralph S. J. Koijen, 2010, Predictive regressions: A presentvalue approach, The Journal of Finance 65, 1439-1471.

Verdinelli, Isabella, and Larry Wasserman, 1995, Computing Bayes factors using a generalization of the Savage-Dickey density ratio, Journal of the American Statistical Association $90,614-618$.

Wachter, Jessica A., 2010, Asset Allocation, Annual Review of Financial Economics 2, 175206.

Wachter, Jessica A., and Missaka Warusawitharana, 2009, Predictable returns and asset allocation: Should a skeptical investor time the market?, Journal of Econometrics 148, $162-178$.

Wright, Jonathan H., 2008, Bayesian Model Averaging and exchange rate forecasts, Journal of Econometrics 146, 329 - 341.

Zellner, Arnold, 1986, On assessing prior distributions and Bayesian regression analysis with $g$-prior distributions, in P.K. Goel, and A. Zellner, eds.: Bayesian Inference and Decision Techniques: Essays in Honour of Bruno de Finetti (North-Holland, Amsterdam, The Netherlands ).

Zellner, Arnold, 1996, An introduction to Bayesian inference in econometrics. (John Wiley and Sons, Inc. New York, NY). 
Table 1: Bayes factors and conditional posterior means

\begin{tabular}{|c|c|c|c|c|c|}
\hline \multirow[b]{2}{*}{$P\left(R^{2}>0.01 \mid H_{1}\right)$} & \multirow[b]{2}{*}{ Bayes factor } & \multicolumn{4}{|c|}{ Posterior Means } \\
\hline & & $\beta$ & $\rho$ & $\mu_{r}$ & $\mu_{x}$ \\
\hline \multicolumn{6}{|c|}{ Panel A: Exact likelihood } \\
\hline 0 & Undefined & 0 & 0.997 & 3.45 & -3.25 \\
\hline 0.05 & 4.13 & 1.07 & 0.989 & 3.77 & -3.35 \\
\hline 0.25 & 6.48 & 1.65 & 0.985 & 3.85 & -3.38 \\
\hline 0.50 & 6.13 & 1.91 & 0.983 & 3.88 & -3.39 \\
\hline 0.99 & 0.01 & 2.06 & 0.982 & 3.90 & -3.40 \\
\hline \multicolumn{6}{|c|}{ Panel B: Conditional likelihood } \\
\hline 0 & Undefined & 0 & 0.998 & 4.48 & -6.83 \\
\hline 0.05 & 2.00 & 0.74 & 0.993 & 3.70 & -5.28 \\
\hline 0.25 & 2.71 & 1.36 & 0.988 & 3.39 & -4.79 \\
\hline 0.50 & 2.56 & 1.66 & 0.985 & 3.11 & -4.78 \\
\hline 0.99 & 0.01 & 1.80 & 0.984 & 2.15 & -5.03 \\
\hline \multicolumn{6}{|c|}{ Panel C: Ordinary least squares } \\
\hline & & 2.97 & 0.973 & 4.49 & -3.54 \\
\hline
\end{tabular}

Notes: The Bayes factor equals the probability of the data $D$ given the predictability model $H_{1}$ divided by the probability of the data given the no-predictability model $H_{0}$ : $p\left(D \mid H_{1}\right) / p\left(D \mid H_{0}\right)$. Bayes factors are reported for various priors on the strength of predictability under $H_{1}$, indexed by $P\left(R^{2}>0.01 \mid H_{1}\right)$ (namely, the prior probability that the population $R^{2}$ exceeds 0.01 , assuming $H_{1}$ ). Posterior means are conditional on $H_{1}$ and are computed for the predictability coefficient $\beta$, the persistence of the dividend-price ratio $\rho$, the mean of the continuously compounded excess return $\mu_{r}$, and the mean of the predictor variable $\mu_{x}$. In Panel $\mathrm{C}, \mu_{r}$ and $\mu_{x}$ equal the sample means. Data are quarterly from $7 / 1 / 1952$ to $3 / 31 / 2009$. 
Table 2: Posterior statistics

\begin{tabular}{|c|c|c|c|c|}
\hline \multirow[t]{2}{*}{$P\left(R^{2}>0.01 \mid H_{1}\right)$} & \multicolumn{4}{|c|}{ Prior probability of return predictability $q$} \\
\hline & 0.20 & 0.50 & 0.80 & 0.99 \\
\hline \multicolumn{5}{|c|}{ Panel A: Posterior probability of predictability $\bar{q}$} \\
\hline 0.05 & 0.51 & 0.80 & 0.94 & 1.00 \\
\hline 0.25 & 0.62 & 0.87 & 0.96 & 1.00 \\
\hline 0.50 & 0.61 & 0.86 & 0.96 & 1.00 \\
\hline 0.99 & 0.00 & 0.01 & 0.05 & 0.54 \\
\hline \multicolumn{5}{|c|}{ Panel B: Posterior mean of predictive coefficient $\beta$} \\
\hline 0.05 & 0.55 & 0.86 & 1.01 & 1.07 \\
\hline 0.25 & 1.02 & 1.43 & 1.59 & 1.65 \\
\hline 0.50 & 1.16 & 1.64 & 1.84 & 1.91 \\
\hline 0.99 & 0.01 & 0.02 & 0.09 & 1.12 \\
\hline \multicolumn{5}{|c|}{ Panel C: Posterior mean of $R^{2}$ (in percentages) } \\
\hline 0.05 & 0.30 & 0.48 & 0.56 & 0.59 \\
\hline 0.25 & 0.59 & 0.83 & 0.92 & 0.95 \\
\hline 0.50 & 0.68 & 0.97 & 1.08 & 1.12 \\
\hline 0.99 & 0.00 & 0.01 & 0.06 & 0.68 \\
\hline
\end{tabular}

Panel D: Difference in CER between optimal and no-predictability strategies

$\begin{array}{lllll}0.05 & 0.38 & 0.84 & 1.10 & 1.20 \\ 0.25 & 0.85 & 1.45 & 1.71 & 1.81 \\ 0.50 & 1.00 & 1.72 & 2.03 & 2.15 \\ 0.99 & 0.00 & 0.00 & 0.02 & 1.67\end{array}$

Notes: The table reports statistics of the posterior distribution averaged over the models $H_{1}$ (predictability) and $H_{0}$ (no predictability). The parameter $q$ denotes the prior probability of $H_{1}$. Statistics are reported for various value of $q$ and for priors on the strength of predictability under $H_{1}$, indexed by $P\left(R^{2}>0.01 \mid H_{1}\right)$ (namely, the prior probability that the population $R^{2}$ exceeds 0.01 , assuming $H_{1}$ ). CER stands for certainty equivalent return and is annualized by multiplying by four. Data are quarterly from $7 / 1 / 1952$ to $3 / 31 / 2009$. 
Table 3: Out of sample certainty equivalent returns (CERs)

\begin{tabular}{|c|c|c|c|c|c|}
\hline \multirow[b]{2}{*}{$P\left(R^{2}>0.01 \mid H_{1}\right)$} & \multicolumn{5}{|c|}{ Prior prob. of return predictability $q$} \\
\hline & 0.01 & 0.20 & 0.50 & 0.80 & 0.99 \\
\hline \multicolumn{6}{|c|}{ Panel A: Comparison with sample mean } \\
\hline \multirow[t]{2}{*}{0.05} & $1.11^{*}$ & $1.08^{*}$ & $1.06^{*}$ & $1.07^{*}$ & $1.07^{*}$ \\
\hline & {$[0.98]$} & {$[1.02]$} & {$[1.03]$} & {$[1.05]$} & {$[1.04]$} \\
\hline \multirow[t]{2}{*}{0.25} & 1.05 & 0.91 & $1.02^{*}$ & $1.08^{*}$ & $1.10^{*}$ \\
\hline & {$[1.07]$} & {$[0.98]$} & {$[0.99]$} & {$[0.99]$} & {$[1.00]$} \\
\hline \multirow[t]{2}{*}{0.50} & 0.85 & $1.09^{*}$ & $1.20^{*}$ & 1.24 & 1.25 \\
\hline & {$[1.08]$} & {$[1.08]$} & {$[1.20]$} & {$[1.32]$} & {$[1.35]$} \\
\hline \multirow[t]{2}{*}{0.99} & $1.17^{*}$ & $1.04^{*}$ & 0.96 & 0.79 & 1.12 \\
\hline & {$[0.99]$} & {$[0.99]$} & {$[1.01]$} & {$[1.05]$} & {$[1.13]$} \\
\hline \multicolumn{6}{|c|}{ Panel B: Comparison with OLS estimates } \\
\hline \multirow[t]{2}{*}{0.05} & 1.06 & 1.03 & 1.02 & 1.02 & 1.03 \\
\hline & {$[1.86]$} & {$[1.85]$} & {$[1.86]$} & {$[1.83]$} & {$[1.83]$} \\
\hline \multirow[t]{2}{*}{0.25} & 1.00 & 0.86 & 0.98 & 1.03 & 1.05 \\
\hline & {$[1.78]$} & {$[1.87]$} & {$[1.86]$} & {$[1.81]$} & {$[1.87]$} \\
\hline \multirow[t]{2}{*}{0.50} & 0.81 & 1.04 & 1.15 & 1.19 & 1.20 \\
\hline & {$[1.91]$} & {$[1.83]$} & {$[1.79]$} & {$[1.80]$} & {$[1.77]$} \\
\hline \multirow[t]{2}{*}{0.99} & 1.12 & 0.99 & 0.92 & 0.74 & 1.07 \\
\hline & {$[1.87]$} & {$[1.87]$} & {$[1.87]$} & {$[1.86]$} & {$[1.85]$} \\
\hline
\end{tabular}


Notes to Table 3: For each year beginning in 1972, the predictive distribution for returns is computed using all data up to that year. Optimal portfolios are computed quarterly to maximize the utility of an agent with constant relative risk aversion equal to 5; these are combined with the actual returns over the following quarter to create out-of-sample returns on the investment strategy. The CER is the riskfree rate of return that generates the same average utility as this series of returns. Panel A reports the CER for the optimal Bayes strategy using the benchmark approach (the benchmark CER) minus the CER for portfolio weights assuming there is no predictability and that the mean and volatility of returns are equal to their sample counterparts. Panel B reports the benchmark CER minus the CER for portfolio weights computed assuming the process for returns is as estimated using OLS. Statistics are reported for various value of $q$ and for priors on the strength of predictability under $H_{1}$ (predictability model), indexed by $P\left(R^{2}>0.01 \mid H_{1}\right)$ (the prior probability that the population $R^{2}$ exceeds 0.01 , assuming $H_{1}$ ) CERs are annualized by multiplying by four. Numbers in brackets report 95 percent critical values, generated using Monte Carlo assuming no return predictability. Starred values are significant at the five percent level using a onetailed test. 
Figure 1: Prior Distribution of the $R^{2}$

Panel A: Probability of predictability $q=1$.

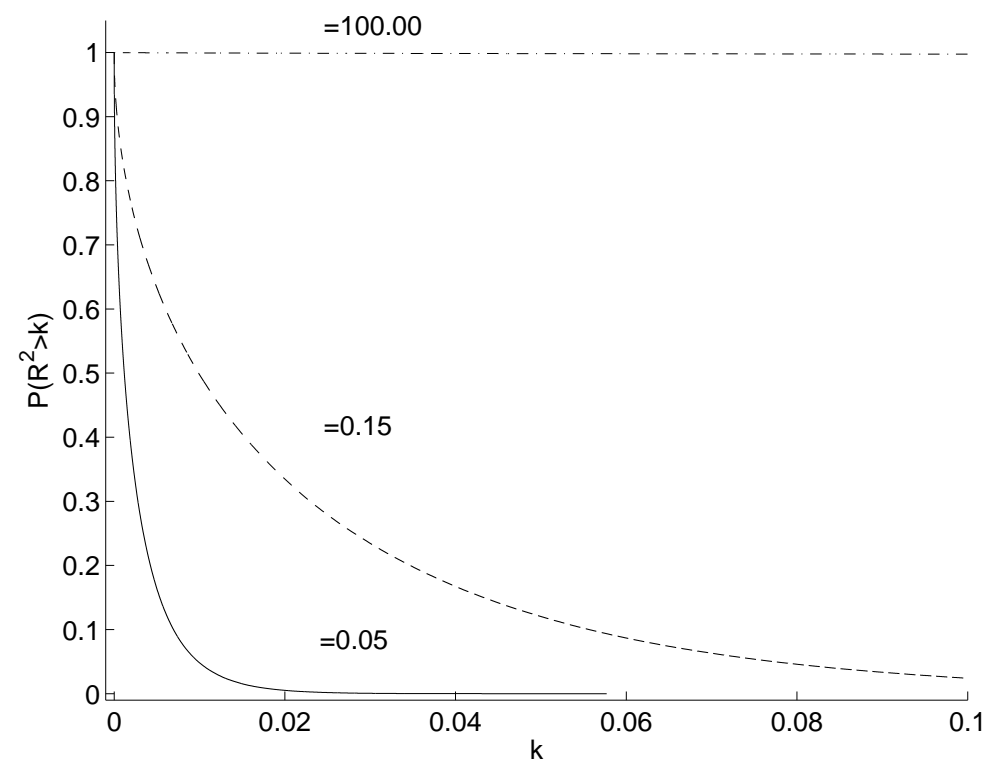

Panel B: Probability of predictability $q=0.5$.

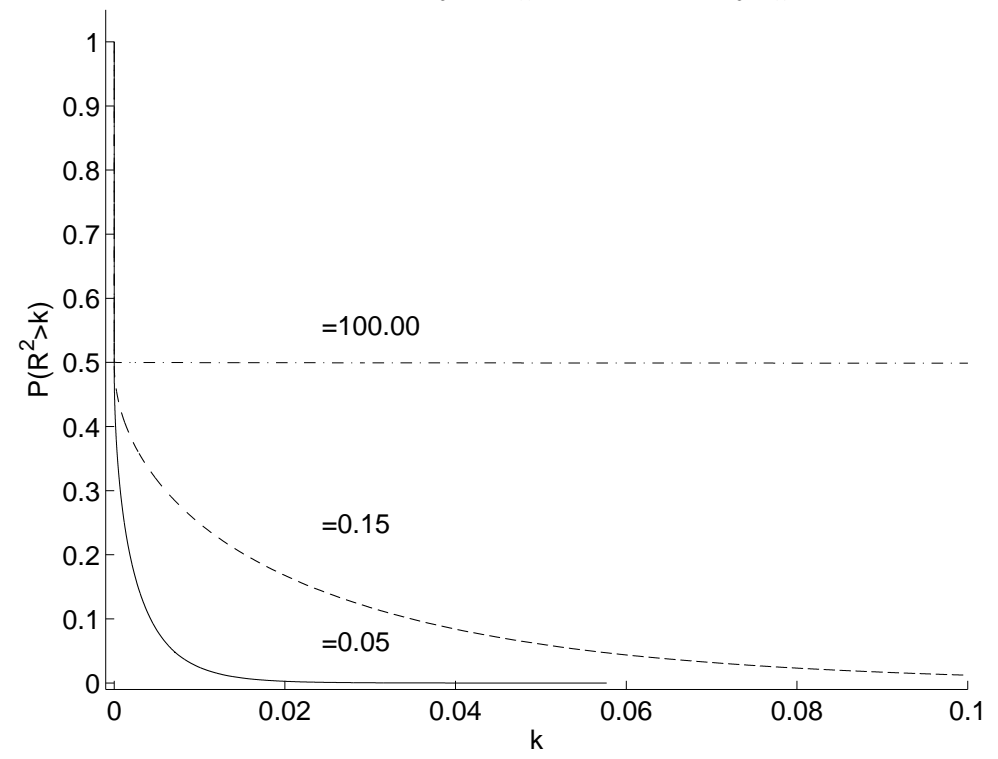

Notes: The figure shows the prior probability that the $R^{2}$ is greater than $k$ for various $k$. This equals one minus the cumulative density function for the prior distribution on the $R^{2}$. Panel A shows the distribution conditional on predictability and Panel B shows the full distribution assuming that the prior probability of predictability is $q=0.5$. The parameter $\sigma_{\eta}$ determines the prior standard deviation of $\beta$ according to the formula $\sigma_{\beta}=\sigma_{\eta} \sigma_{x}^{-1} \sigma_{u}$, where $\sigma_{x}$ is the standard deviation of the predictor variable and $\sigma_{u}$ is the standard deviation of the shock to returns. 
Figure 2: Posterior Distribution of the $R^{2}$
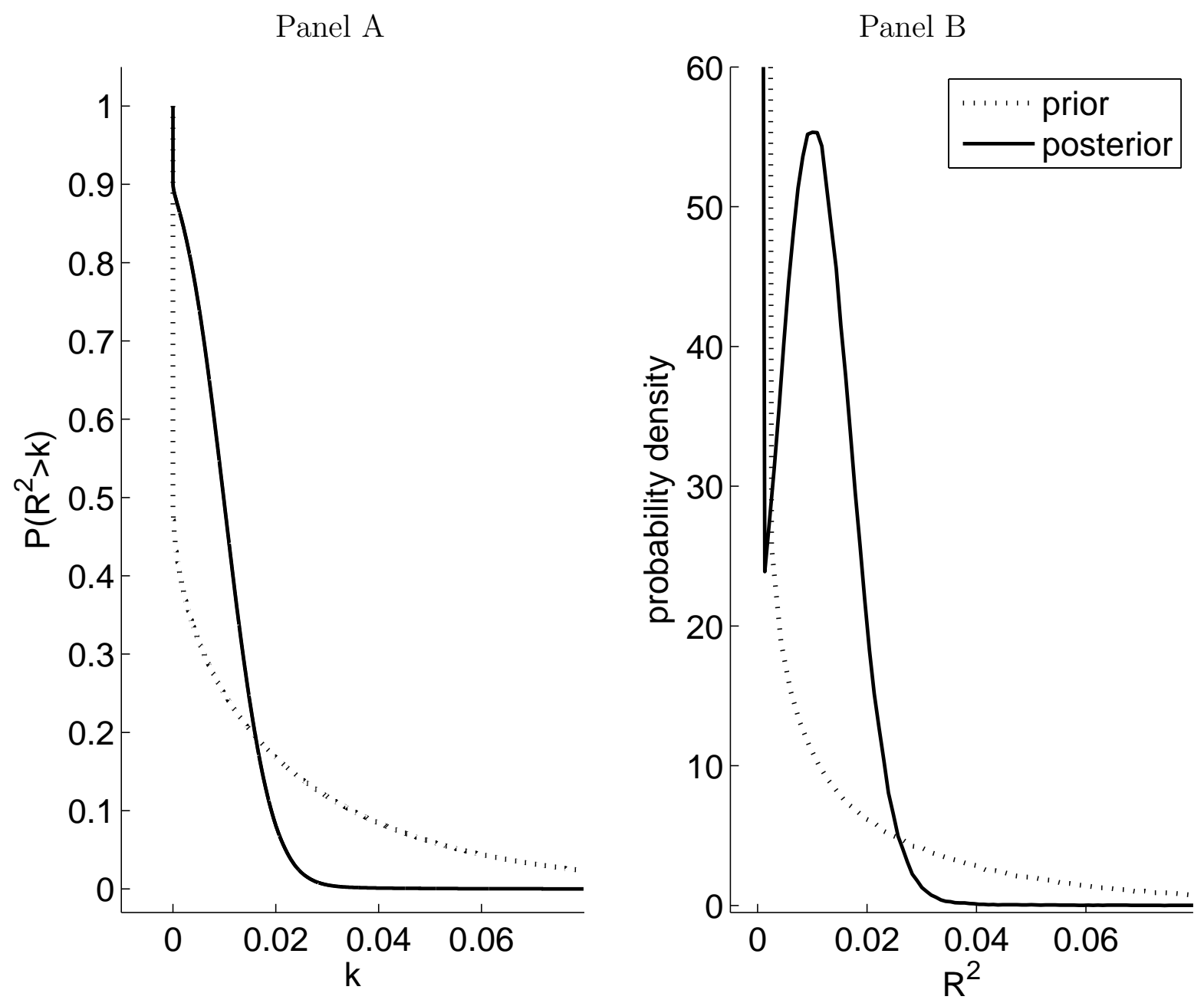

Notes: Panel A shows the prior and posterior probabilities that the $R^{2}$ will be greater than $k$ for various $k$. Panel $\mathrm{B}$ shows the prior and posterior density functions of the $R^{2}$. Priors are such that $P\left(R^{2}>0.01 \mid H_{1}\right)$ (the probability that the $R^{2}$ exceeds $1 \%$ conditional on predictability) equals 0.5 and $q$ (the prior probability of predictability) also equals 0.5. Data are quarterly from 7/1/1952 to 3/31/2009. 
Figure 3: The log dividend-price ratio

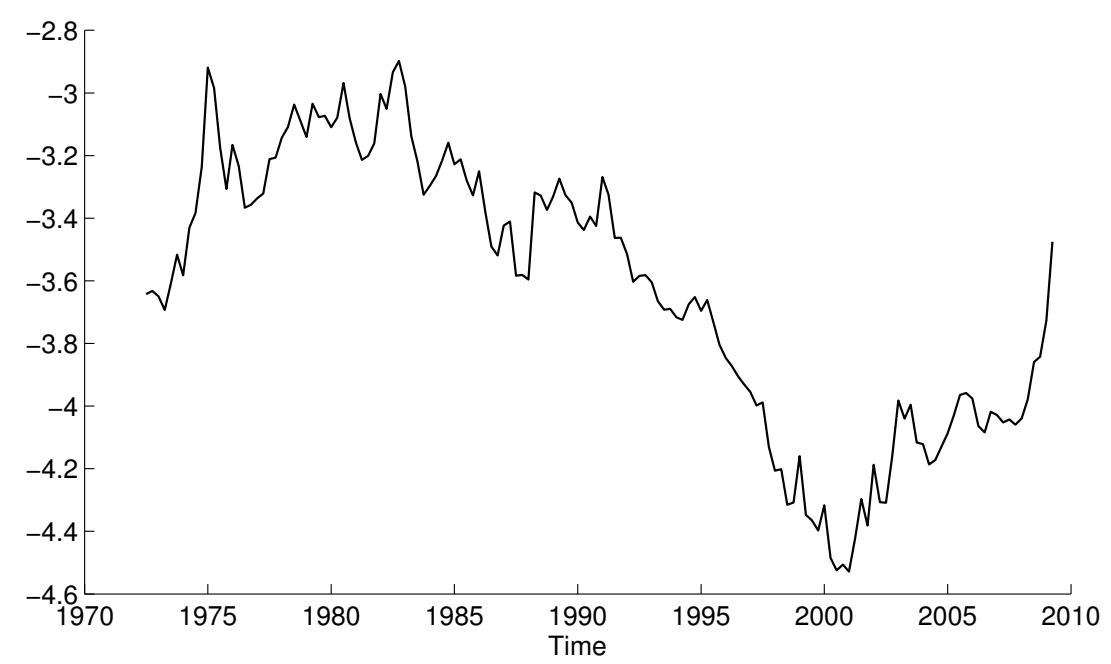

Notes: The figure shows quarterly observations on the log of the dividend-price ratio, computed by dividing the dividend payout over the previous 12 months by the current price. Prices and dividends are for the CRSP value-weighted index. 
Figure 4: The Bayes factor and posterior probability of return predictability

Panel A: Posterior probability of predictability, $\bar{q}$

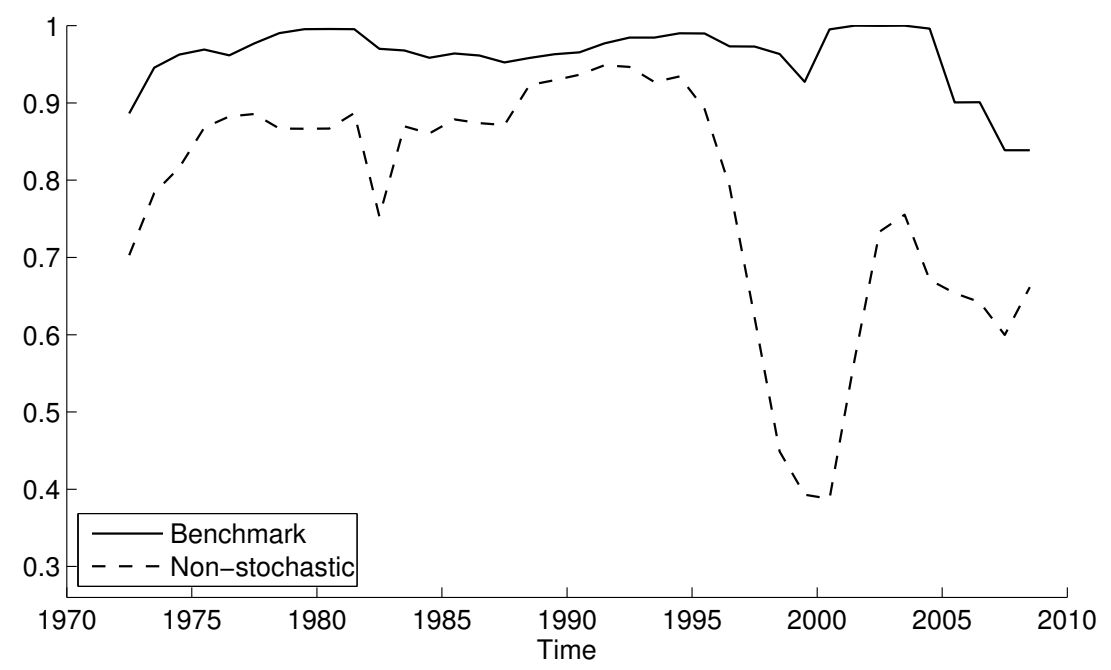

Panel B: Log Bayes factor

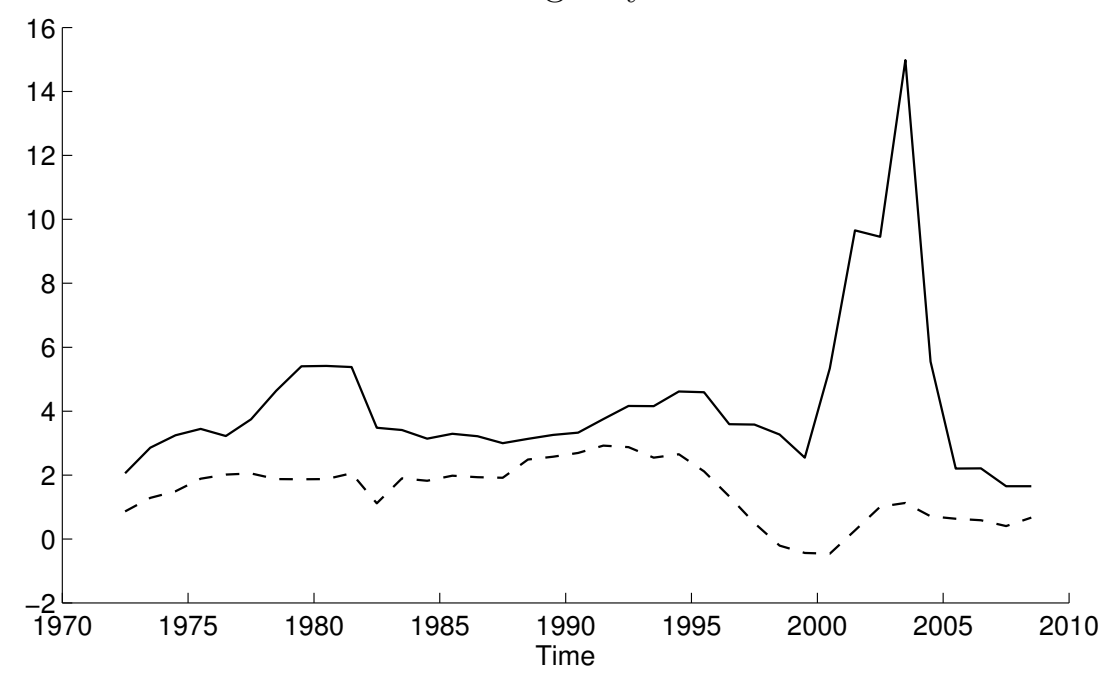

Notes: Panel A shows the posterior probability of $H_{1}$ (the predictability model), assuming a prior probability of 0.5. Panel B shows the log Bayes factor, equal to the log probability of the data given the predictability model $H_{1}$ minus the log probability of the data given the no-predictability model $H_{0}$. Both panels assume $P\left(R^{2}>0.01 \mid H_{1}\right)$ (namely, the prior probability that the population $R^{2}$ exceeds 0.01 , given $H_{1}$ ) equals 0.5 . The Bayes factor and the posterior probability are computed using quarterly data beginning in 7/1/1952 and ending at the time shown on the $x$-axis. The solid line shows results for the benchmark specification. The dashed line shows results for the case of a non-stochastic regressor. 
Figure 5: Posterior means of $\beta$ and $\rho$ over time.

Panel A: Posterior mean of predictive coefficient $\beta$

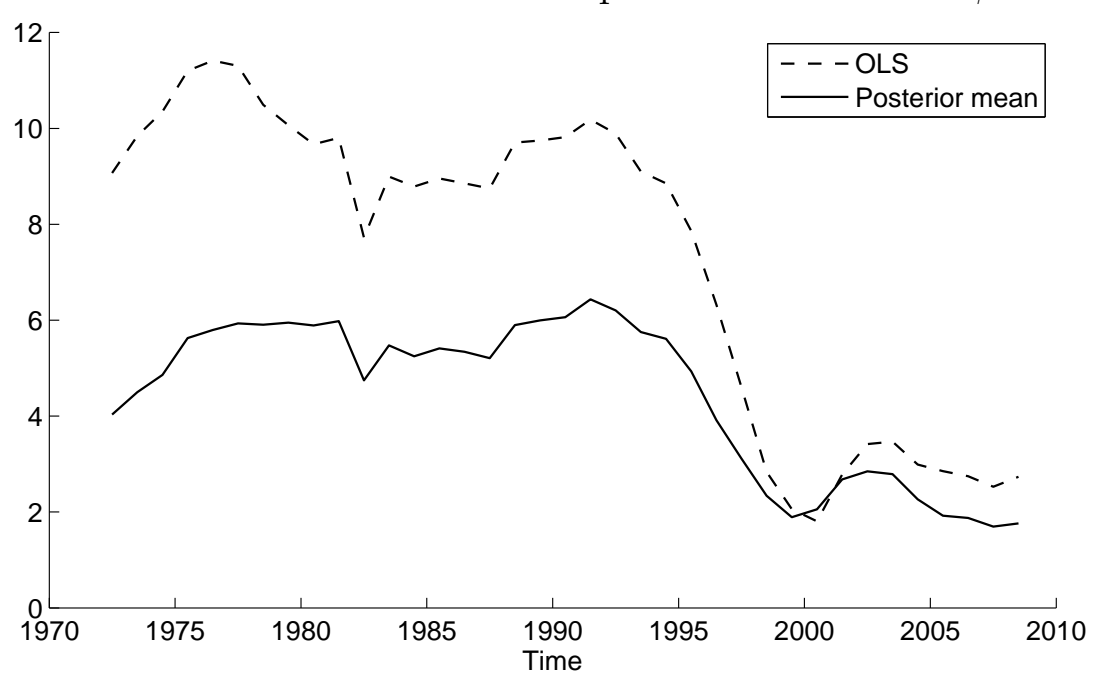

Panel B: Posterior mean of autoregressive coefficient $\rho$

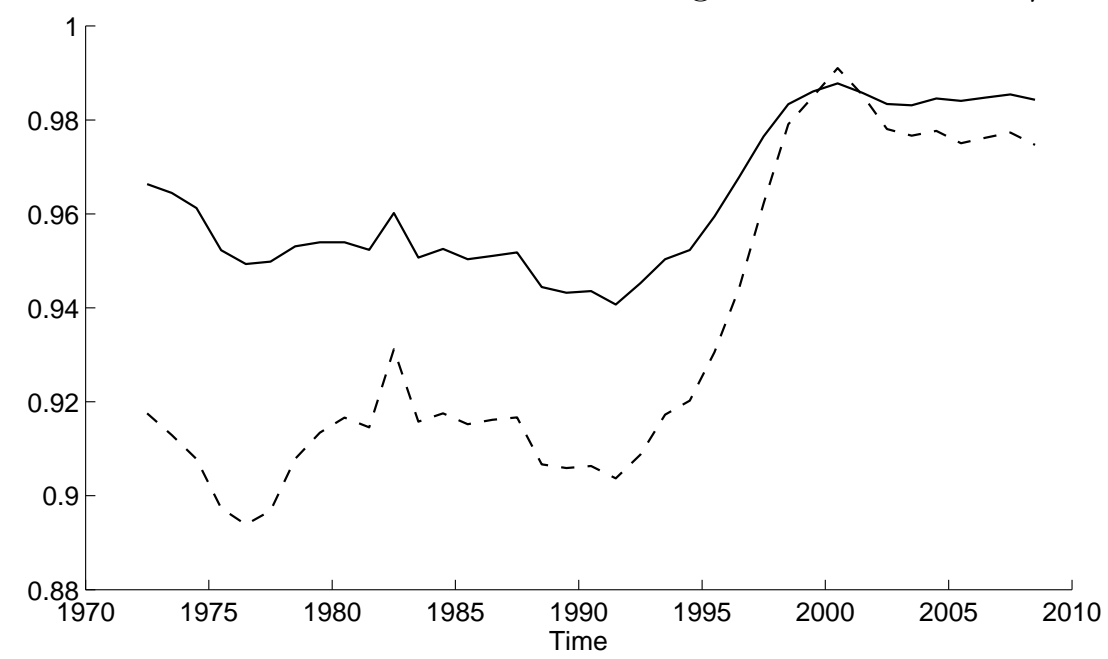

Notes: Panel A shows the posterior mean of $\beta$ under the benchmark specification (solid line) and the OLS estimate of $\beta$ (dashed line) using data beginning in 7/1/1952 and ending at the time shown on the $x$-axis. Panel B shows analogous results for $\rho$, the autoregressive coefficient on the dividend-price ratio. The posterior distributions are computed assuming $q$ (the prior probability that returns are predictable) equal to 0.50 , and assuming $P\left(R^{2}>0.01 \mid H_{1}\right)$ (the prior probability that the population $R^{2}$ exceeds 0.01 , given $H_{1}$ ) also equal to 0.5 . 
Figure 6: Posterior probabilities implied by different methods

Panel A: Benchmark prior with the exact and conditional likelihoods

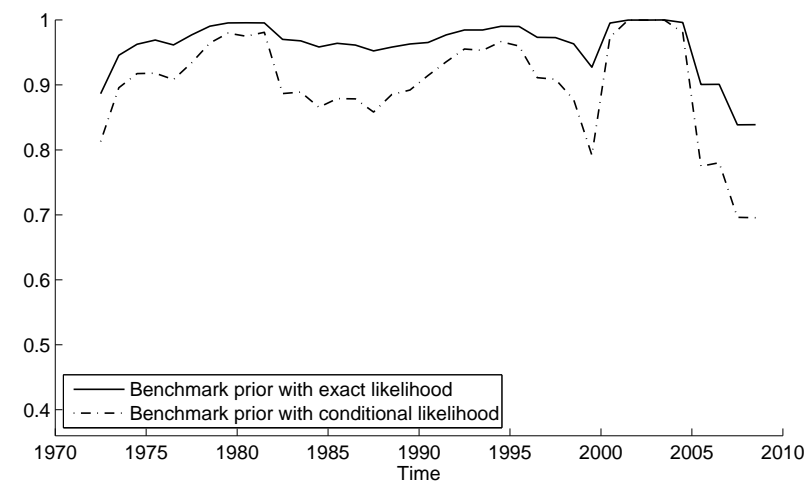

Panel B: Benchmark and empirical Bayes priors with conditional likelihood

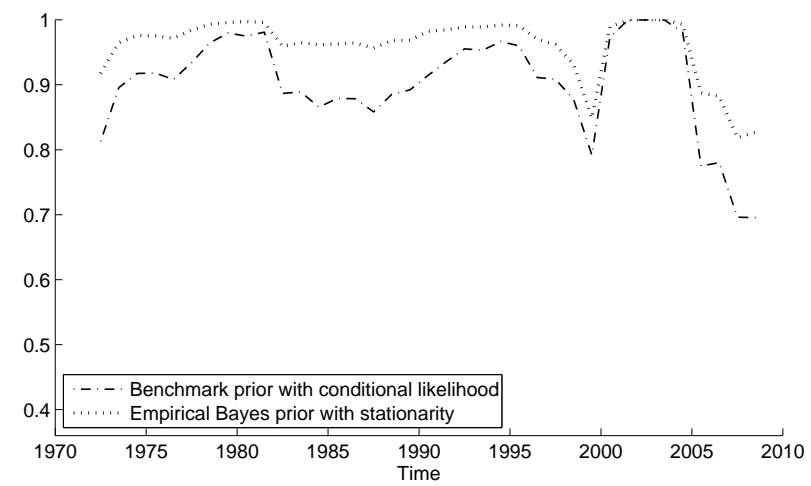

Panel C: Empirical Bayes priors with and without stationarity

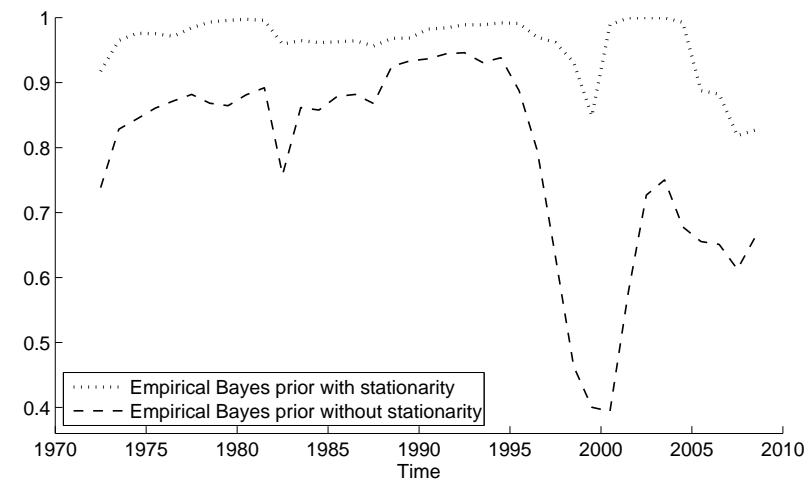

The figures show the posterior probability of return predictability (see Figure 4 for more details). Panel A compares our benchmark specification "Exact likelihood", with a specification that uses the conditional likelihood but keeps the prior the same. Panel B compares this latter specification with one that uses the empirical Bayes prior but keeps everything else the same; note both specifications use the conditional likelihood. Panel $\mathrm{C}$ compares results from the conditional likelihood and empirical Bayes prior assuming stationarity with results from this same specification without assuming stationarity. 
Figure 7: The conjugate prior and posterior with training samples of varying lengths

Panel A: Prior distributions for $\beta$

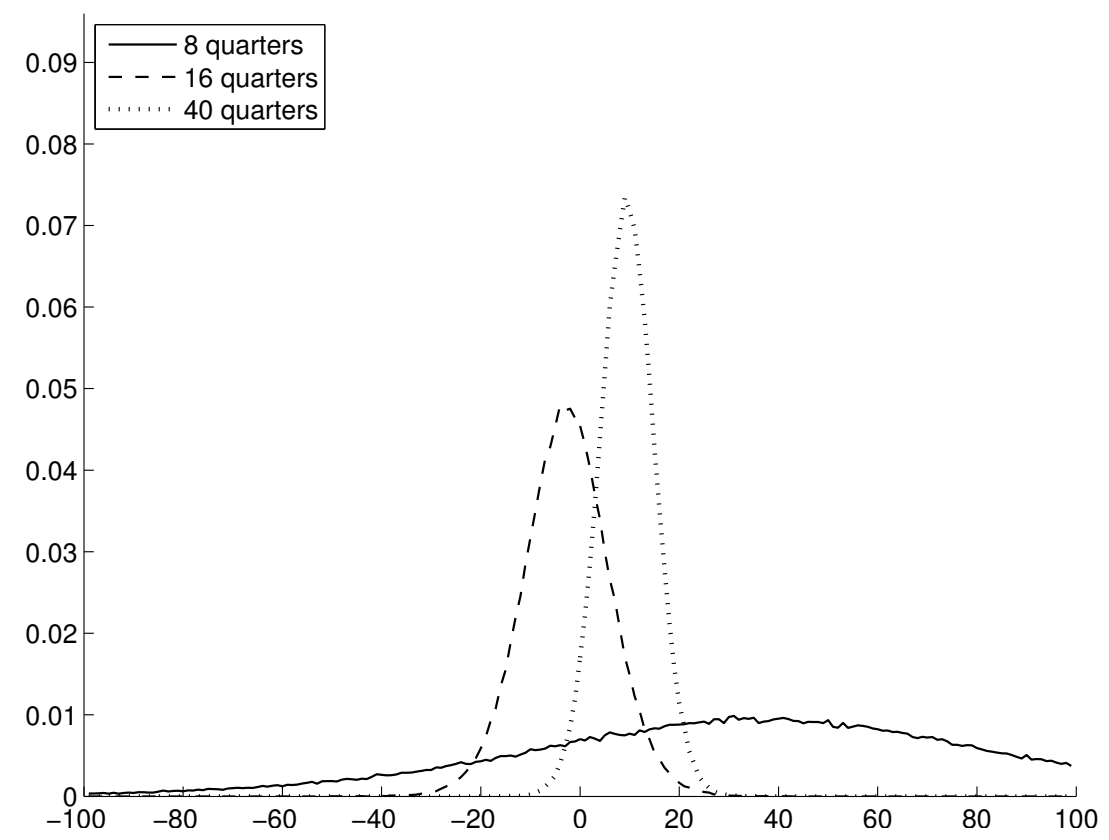

Panel B: Posterior probabilities of predictability

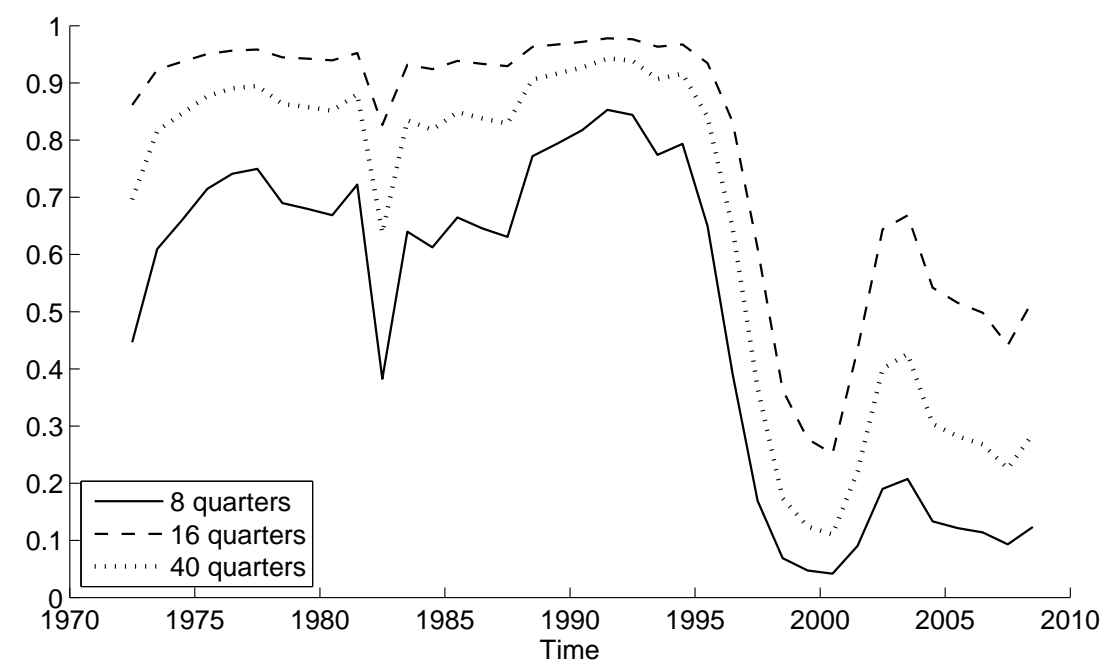

Notes: Panel A shows the prior distribution for $\beta$ assuming training samples of different lengths. Panel B shows the posterior probabilities of predictability. The training samples begin in 7/1/1952 and last for the number of quarters given in the legend. The posterior probabilities are computed using the remaining data, ending at the time shown on the $x$ axis. 
Figure 8: Portfolio weights for the benchmark approach and implied by sample moments

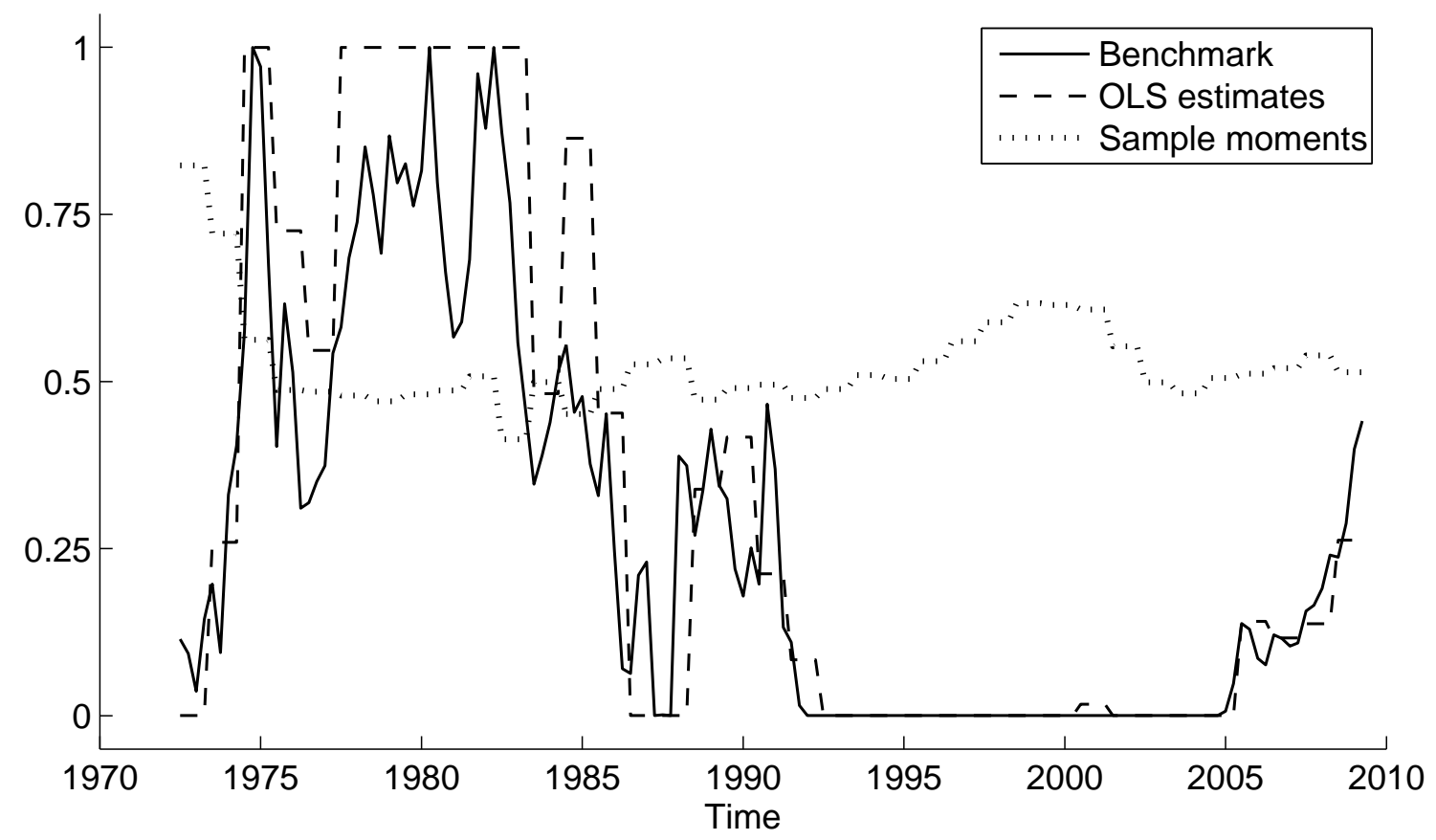

Notes: The figure shows the time series of weights in the risky asset using the benchmark approach and assuming $q=0.50$ and $P\left(R^{2}>0.01 \mid H_{1}\right)=0.50$. The figure also shows the time series of weights assuming that parameters estimated by OLS are known with certainty, as well as the time series of weights computed assuming that returns are not predictable, but that the resulting moments are known with certainty (sample moments). Data are quarterly beginning in $7 / 1 / 1952$ and ending at the time shown on the $x$-axis. 
Figure 9: The Bayes factor and posterior probability of return predictability for the yield spread

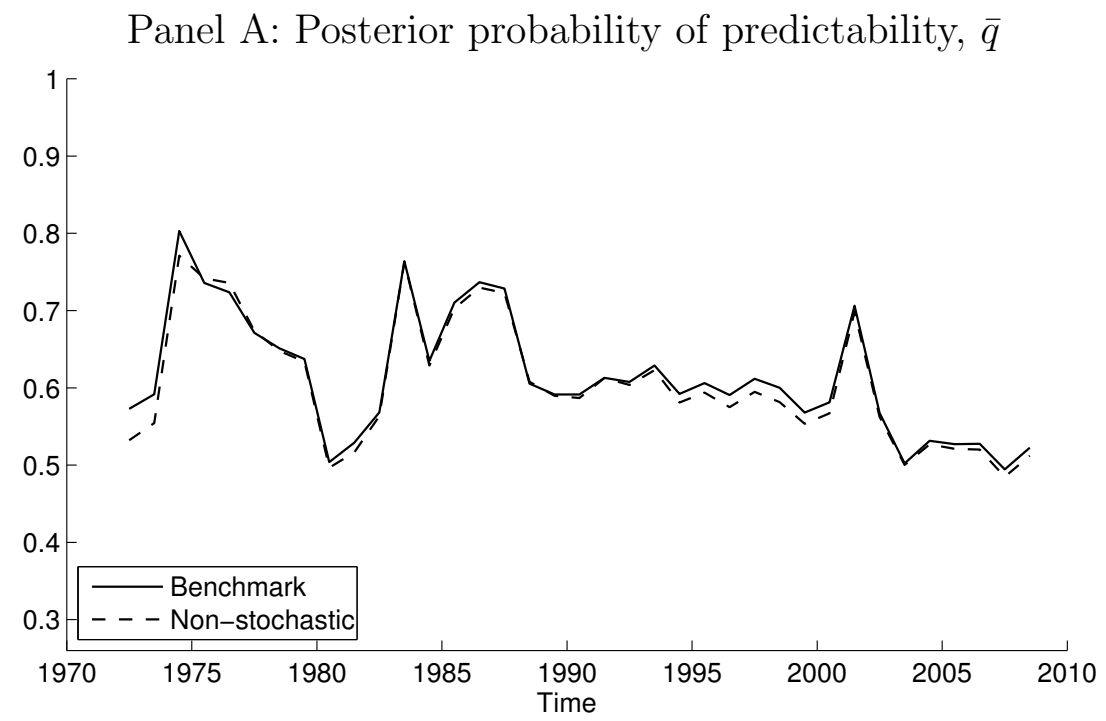

Panel B: Log Bayes Factor

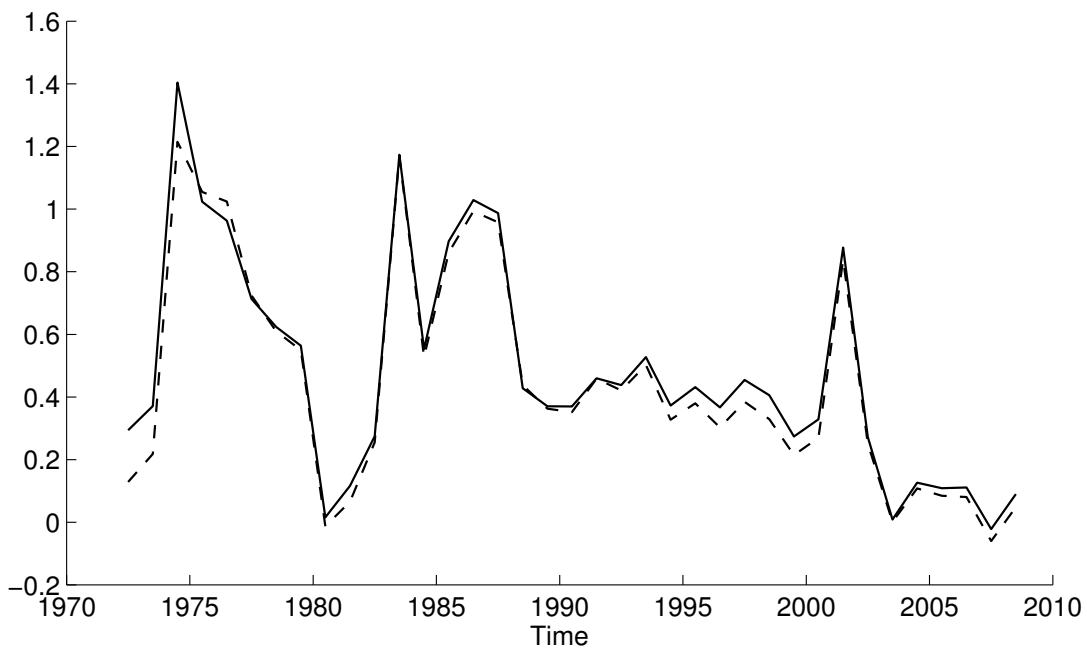

Notes: Panel A assumes the posterior probability of predictability and Panel B shows the log Bayes factor, assuming the predictive variable is the yield spread, namely the continuouslycompounded yield on the five-year zero coupon bond less the continuously-compounded yield on the 3-month Treasury bill. The solid line shows results for the benchmark specification. The dashed line shows results assuming a non-stochastic regressor. 